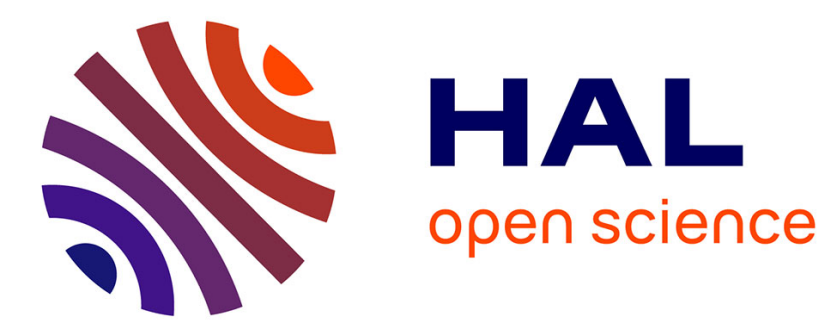

\title{
Comprehensive Multifractal Analysis of Turbulent Velocity Using the Wavelet Leaders
}

Bruno Lashermes, Stéphane G. Roux, Patrice Abry, Stéphane Jaffard

\section{To cite this version:}

Bruno Lashermes, Stéphane G. Roux, Patrice Abry, Stéphane Jaffard. Comprehensive Multifractal Analysis of Turbulent Velocity Using the Wavelet Leaders. 2007. ensl-00195451

\section{HAL Id: ensl-00195451 \\ https://hal-ens-lyon.archives-ouvertes.fr/ensl-00195451}

Preprint submitted on 11 Dec 2007

HAL is a multi-disciplinary open access archive for the deposit and dissemination of scientific research documents, whether they are published or not. The documents may come from teaching and research institutions in France or abroad, or from public or private research centers.
L'archive ouverte pluridisciplinaire HAL, est destinée au dépôt et à la diffusion de documents scientifiques de niveau recherche, publiés ou non, émanant des établissements d'enseignement et de recherche français ou étrangers, des laboratoires publics ou privés. 


\title{
Comprehensive Multifractal Analysis of Turbulent Velocity Using the Wavelet Leaders
}

\author{
Bruno Lashermes ${ }^{1}$, Stéphane G. Roux ${ }^{2}$, Patrice Abry ${ }^{2}$, and Stéphane Jaffard ${ }^{3}$ \\ 1 St Anthony Falls Laboratory, Dept. of Civil Engineering, University of Minnesota and National Center for Earth-surface \\ Dynamics \\ 2 3rd Ave S - Minneapolis, MN, 55414 - USA \\ 2 Laboratoire de Physique, École Normale Supérieure de Lyon and CNRS \\ 46, allée d'Italie - 69007 Lyon - France \\ 3 Laboratoire d'Analyse et de Mathématiques Appliquées, Université Paris XII \\ 61, avenue du Général de Gaulle - 94010 Créteil - France
}

the date of receipt and acceptance should be inserted later

\begin{abstract}
The multifractal framework relates the scaling properties of turbulence to its local regularity properties through a statistical description as a collection of local singularities. The multifractal properties are moreover linked to the multiplicative cascade process that creates the peculiar properties of turbulence such as intermittency. A comprehensive estimation of the multifractal properties of turbulence from data analysis, using a tool valid for all kind of singularities (including oscillating singularities) and mathematically well-founded, is thus of first importance in order to extract a reliable information on the underlying physical processes. The wavelet leaders yield a new multifractal formalism which meets all these requests. This paper aims at describing it and at applying it to experimental turbulent velocity data. After a detailed discussion of the practical use of the wavelet leader based multifractal formalism, the following questions are carefully investigated: (1) What is the dependence of multifractal properties on the Reynolds number? (2) Are oscillating singularities present in turbulent velocity data? (3) Which multifractal model does correctly account for the observed multifractal properties? Results from several data set analysis are used to discuss the dependence of the computed multifractal properties on the Reynolds number but also to assess their common or universal component. An exact though partial answer (no oscillating singularities are detected) to the issue of the presence of oscillating singularities is provided for the first time. Eventually an accurate parameterization with cumulants exponents up to order 4 confirms that the log-normal model (with $c_{2}=-0.025 \pm 0.002$ ) correctly accounts for the universal multifractal properties of turbulent velocity.
\end{abstract}

PACS. XX.XX.XX No PACS code given

\section{Introduction}

Turbulent velocity and multifractal description. The streamwise component of turbulent flow velocity spatial fields is known to exhibit a main feature: very irregular fluctuations over a large range of scales, whose statistical moments furthermore behave within the so-called inertial scale range like power laws with respect to the scale $a$ :

$$
<\left|v\left(x_{0}+a\right)-v\left(x_{0}\right)\right|^{q}>\sim a^{\zeta(q)}
$$

where $<$. $>$ denotes the spatial averaging over the locations $x_{0}$. The $\zeta(q)$ are called the scaling exponents. Characterization and understanding of the observed scaling properties play a central role in the theoretical description of turbulence, following the seminal work by Kolmogorov in 1941 [1] in which were predicted linear scaling exponents: $\zeta(q)=q / 3$. This prediction was actually lacking of consistency and has been refined by Obukhov and Kol- mogorov in $1962[2,3]$ who predicted a (quadratic) nonlinear behavior of the scaling exponents. The non-linear behavior of the $\zeta(q)$ was confirmed by various experimental results and other $\zeta(q)$ models have been proposed (see [4] for an overview). The non-linear behavior of the $\zeta(q)$ received an interpretation through the existence of an underlying multiplicative cascade structure of the energy dissipation field $[5,6]$ (according to the early heuristics by Richardson [7]) which results in a multiplicative cascade structure of the velocity fluctuations [8].

The multifractal (MF) framework relates the scaling properties to the local regularity properties by describing a turbulent velocity field as a collection of local singularities (roughly speaking simple singularities: $\left|x-x_{0}\right|^{h}$ or oscillating singularities: $\left|x-x_{0}\right|^{h} \sin \left(1 /\left|x-x_{0}\right|^{\beta}\right)$ which regularity is characterized by the exponent $h$ ) which are distributed on interwoven fractal sets. The local regularity receives a proper definition with the Hölder exponent and 
the MF analysis aims at characterizing the spatial distribution of Hölder exponents with the so-called singularity spectrum $D(h)$. Though many MF models of turbulence implicitly involve simple singularities only and in spite of several works [9-12], the existence of oscillating singularities in turbulent velocity data remains an open issue.

Practical multifractal analysis. One of the main goals of numerical analysis of experimental turbulent velocity data is the measurement of the MF properties, i.e., the singularity spectrum $D(h)$, for instance to discriminate between the various proposed MF models. The singularity spectrum can be (at least partially) computed from real data using a MF formalism (first introduced in the mid 80's [13]) which relates the $\zeta(q)$ to $D(h)$ through a Legendre transform.

Development of the wavelet transforms allowed real enhancements in various fields of signal processing [14], for instance the improvement of the MF formalisms [15-17]. The MF formalisms based on either increment or wavelet coefficients suffer from a common drawback: the $\zeta(q)$ with negative orders $q$ and hence the right part of $D(h)$ cannot be computed. The WTMM (Wavelet Transform Modulus Maxima) MF formalism has been introduced in order to overcome this failure $[15,18,19]$. This formalism has been checked to compute the whole singularity spectrum on a selection of synthetic MF processes with simple singularities but does not receive a theoretical backing yet.

Moreover, in spite of several works $[11,12]$ the effect of oscillating singularities on the use of MF formalisms is poorly understood but is known to possibly result in the failure of the wavelet coefficient based MF formalisms [17].

Wavelet leaders. Wavelet leaders (WL) are multiresolution coefficients defined from a specific wavelet transform (the discrete wavelet transform) which have recently been introduced in order to define a MF formalism allowing a comprehensive MF analysis (measurement of the whole singularity spectrum) backed by precise mathematical results $[20,21]$. WL provide a characterization better suited to the derivation of a MF formalism than wavelet coefficients and with a wider range of validity. As a consequence the WL based MF formalism is proven to be valid for functions with oscillating singularities for which the one based on the wavelet coefficients is known to fail.

This new MF formalism has been implemented and numerically characterized on synthetic MF data in [22-24]. Its application to experimental turbulent velocity data is the aim of this paper and we show that the unique properties of the WL based MF formalism (complete MF analysis relying on theoretical basis and validity for all kind of singularities) indeed bring interesting and new results on the MF description of turbulent velocity.

Outline. This paper is organized as follows. Section 2 provides a presentation of the MF framework for functions. Precise definitions of pointwise regularity, Hölder exponent and singularity spectrum are displayed before an overview of the classical ways to perform a practical
MF analysis: the MF formalism and the cumulant analysis. Section 3 discusses the important choice of multiresolution coefficients, introduces the WL and the derived MF formalism. Its validity is discussed and compared to former MF formalisms and illustrated using toy-examples. Section 4 discusses the use of MF description in the turbulence field as well as the underlying assumptions. Practical MF analysis of several experimental turbulent velocity data sets using the WL is then performed in Section 5 and all different steps are carefully discussed. Results from experimental data analysis are compared to previous results and conclusions about the MF properties of turbulent velocity are eventually drawn and discussed. Final conclusions are given in Section 6.

\section{Multifractal framework}

The MF framework is a statistical description of a function $f$ through its pointwise (or local) regularity properties (see $[17,24]$ for a thorough introduction).

\subsection{Multifractal analysis}

\subsubsection{Hölder exponent}

The pointwise regularity of a function $f$ at point $x_{0}$ is properly defined by the Hölder exponent $h\left(x_{0}\right)$ (the following definition actually holds for $h<1$ only but easily extends to $h \geq 1$, see [24]):

$$
h\left(x_{0}\right)=\operatorname{Sup}\left\{\alpha: f \in C^{\alpha}\left(x_{0}\right)\right\},
$$

where

$$
f \in C^{\alpha}\left(x_{0}\right) \text { if }\left|x-x_{0}\right| \leq \epsilon,\left|f(x)-f\left(x_{0}\right)\right| \leq C\left|x-x_{0}\right|^{\alpha} .
$$

The value of the Hölder exponent is interpreted as follows: the closer to $0 h\left(x_{0}\right)$ is, the more irregular (or singular) at point $x_{0}$ the function is. In contrast, a larger value of $h\left(x_{0}\right)$ is related to a smoother (more regular) behavior at $x_{0}$.

\subsubsection{Simple and oscillating singularities}

A specific value $h\left(x_{0}\right)$ for the Hölder exponent at point $x_{0}$ does not necessarily imply a local power law behavior ( simple singularity or cusp):

$$
\left|f(x)-f\left(x_{0}\right)\right| \simeq b\left|x-x_{0}\right|^{h\left(x_{0}\right)}
$$

when $x \rightarrow x_{0}$. Other local singular behaviors indeed result in the same value for the Hölder exponent. This is the case, for instance, for an oscillating singularity (or chirp):

$$
\left|f(x)-f\left(x_{0}\right)\right| \simeq b\left|x-x_{0}\right|^{h\left(x_{0}\right)} \sin \left(\frac{1}{\left|x-x_{0}\right|^{\beta\left(x_{0}\right)}}\right)
$$

when $x \rightarrow x_{0} ; \beta\left(x_{0}\right)$ is called the oscillating exponent.

The local regularity properties cannot thus be extensively characterized with simple singularities only and a comprehensive MF analysis has to take into account all possible local behaviors such as oscillating singularities. 


\subsubsection{Singularity spectrum}

The points $x_{0}$ associated to a specific Hölder exponent value $h$ are distributed on interwoven fractal subsets $E(h)$ :

$$
E(h)=\left\{x_{0}: h\left(x_{0}\right)=h\right\} .
$$

In that case, the MF description provides an efficient characterization of MF functions through a hierarchical classification of the subsets $E(h)$ using their Hausdorff dimension (see for instance [25]):

$$
D(h)=\operatorname{Dim}_{\mathrm{H}} E(h) .
$$

(By convention $\operatorname{Dim}_{H} E=-\infty$ if $E$ is the empty set.)

The function $D(h)$ is called the singularity spectrum and its estimation is the goal of MF analysis. The singularity spectrum has been shown to be a relevant quantity to characterize stochastic processes in nature (for instance in turbulence, see [4]) since it is related to the underlying statistical structure of data fluctuations.

Monofractal functions are a specific subclass of $\mathrm{MF}$ functions for which the Hölder exponent takes a unique value $H$ at every point $x_{0}$. The singularity spectrum then reduces to a single point: $D(h)=1$ if $h=H$ and $D(h)=-\infty$ if $h \neq H$. A well known example of monofractal processes is the fractional Brownian motion which has been proposed by Kolmogorov in 1941 [26] to model turbulent velocity, with $H=1 / 3$. In contrast a function is multifractal if the Hölder exponent can take different values: its singularity spectrum thus takes finite values on an interval $\left[h_{\min }, h_{\max }\right]$ with $h_{\max }>h_{\min }$. For most MF functions the singularity spectrum $D(h)$ is a concave function which is bell-shaped (see Fig. 7 for instance). The spectrum $D(h)$ then reaches its maximum at an abscissa $h_{0}$ and the left (right) part of the singularity spectrum is defined in this paper as the part of $D(h)$ for which $h \leq h_{0}$ $\left(h \geq h_{0}\right)$.

\subsection{Multifractal formalisms}

\subsubsection{Structure functions and scaling exponents}

MF analysis is performed on data with specific tools, the MF formalisms. The singularity spectrum $D(h)$ is estimated from the statistics of the local fluctuations $c\left(x_{0}, a\right)$ of the function at different scales $a$ and at different locations $x_{0}$. The archetypal choice [13] for the local fluctuations (or multi-resolution coefficients) $c\left(x_{0}, a\right)$ is the increment coefficients:

$$
c\left(x_{0}, a\right)=\delta\left(x_{0}, a\right)=f\left(x_{0}+a\right)-f\left(x_{0}\right) .
$$

If the Hölder exponent at $x_{0}$ is $h$, then

$$
\left|c\left(x_{0}, a\right)\right| \sim a^{h} .
$$

The structure functions $S(q, a)$ are defined as estimates (by space-averaging) of the $q$-th statistical moments of the absolute value of the $c\left(x_{0}, a\right)$ at scale $a$ :

$$
S(q, a)=\frac{1}{n(a)} \sum_{x_{0}}\left|c\left(x_{0}, a\right)\right|^{q}
$$

where $n(a)$ is the number of coefficients $c\left(x_{0}, a\right)$ available at scale $a$.

Structure functions behave like power laws for $\mathrm{MF}$ functions when $a \rightarrow 0$ :

$$
S(q, a) \sim a^{\zeta(q)}
$$

defining the usual scaling exponents $\zeta(q)$, indexed by order $q$.

\subsubsection{Multifractal formalisms}

MF formalisms state that scaling exponents $\zeta(q)$ relate to the singularity spectrum $D(h)$ through a Legendre transform:

$$
D(h)=1+\min _{q}[q h-\zeta(q)] .
$$

The Legendre transform link between the scaling exponents and the singularity spectrum can be heuristically understood as follows (this is not a formal proof). Since the Hausdorff dimension of the subset $E(h)$ is $D(h)$, the number of multi-resolution coefficients, whose typical size is the scale $a$, needed to cover $E(h)$ is proportional to $a^{-D(h)}$ when $a \rightarrow 0$. Furthermore one can assume that $n(a) \sim a^{-1}$. Since (9) holds when $a \rightarrow 0$, the contribution of a specific Hölder exponent $h$ to $S(q, a)$ is thus:

$$
\sim a^{1} a^{-D(h)} a^{q h}=a^{1+q h-D(h)} .
$$

For a given order $q$ the contribution of the Hölder exponent value $h$ such that $1+q h-D(h)$ is minimal becomes predominant when $a \rightarrow 0$ :

$$
S(q, a) \sim a^{1+\min _{h}(q h-D(h))},
$$

leading to the identification:

$$
\zeta(q)=1+\min _{h}[q h-D(h)] .
$$

In turns, since the inverse of the Legendre transform of a concave function is the Legendre transform, one may expect (12) to hold. Note that the validity of (9) is a key argument in this derivation. Alternative MF formalisms have to be based on multi-resolution coefficients which satisfy this requirement. The issue of their choice will be addressed in Section 3.1.

Note that Eq. (15) implies a linear behavior of the scaling exponents for monofractal functions (cf. Section 2.1.3): $\zeta(q)=q H$ and a non-linear behavior for MF functions.

The MF formalism is valid if the Legendre transform of the scaling exponents is equal to the singularity spectrum. Eq. (12) is indeed not a theoretical result and the Legendre transform of the scaling exponents may depart from the singularity spectrum. The validity of a MF formalism actually depends on the specific choice of multi-resolution coefficients $c\left(x_{0}, a\right)$. This is one of the key-point discussed all along this paper. 


\subsubsection{Cumulant analysis}

Pertinent parameters for classification of singularity spectrum $D(h)$ are provided by the so-called cumulant analysis method, which is a tool used in turbulence [27,28] and in other fields $[29,30]$. This method provides estimates of the parameters $c_{p}$ of the Taylor series expansion of $\zeta(q)$ for $q \rightarrow 0^{1}$ :

$$
\zeta(q)=\sum_{p \geq 1} \frac{c_{p}}{p !} q^{p} .
$$

The estimation of the $c_{p}$ is performed by computing (with spatial averaging) the cumulants $C(p, a)$ of order $p$ of the logarithm of the absolute value of multi-resolution coefficients $c\left(x_{0}, a\right)$ at a given scale $a$. The cumulants $C(p, a)$ are linear functions of $\ln a$ for MF functions when $a \rightarrow 0$. For instance for $p=1$ and 2 :

$$
\begin{gathered}
C(1, a)=\frac{1}{n(a)} \sum_{x_{0}} \ln \left|c\left(x_{0}, a\right)\right| \simeq b_{1}+c_{1} \ln a \\
C(2, a)=\frac{1}{n(a)} \sum_{x_{0}}\left[\ln \left|c\left(x_{0}, a\right)\right|-C(1, a)\right]^{2} \simeq b_{2}+c_{2} \ln a .
\end{gathered}
$$

Linear regressions of $C(p, a)$ versus $\ln a$ thus allow the estimation of the $c_{p}$, called the cumulant exponents.

The cumulant analysis is an alternative way of performing MF analysis, whose main interest is to provide a concise parameterization of the singularity spectrum $D(h)$. If the process under analysis is monofractal then $c_{1}=H \neq 0$ and $c_{p}=0$ for $p>1$. A non-zero value for $c_{2}$ explicitly establishes the multifractal (vs. monofractal) nature of the data and the parameter $c_{2}$ (also called the intermittency coefficient) is used to characterize the degree of multifractality. Indeed, a quadratic approximation of the scaling exponents: $\zeta(q) \simeq c_{1} q+c_{2} q^{2} / 2($ when $q \rightarrow 0)$ which corresponds to a quadratic approximation of the singularity spectrum: $D(h) \simeq 1+\frac{\left(h-c_{1}\right)^{2}}{2 c_{2}}\left(\right.$ when $\left.h \rightarrow c_{1}\right)$ is a commonly used MF model (see Section 4.1).

\section{Wavelet leader based multifractal formalism}

\subsection{Choice of multi-resolution coefficients}

The specific choice of the multi-resolution coefficients $c\left(x_{0}, a\right)$ plays a central role for the validity of MF formalisms. wavelet transforms (see [14] for an overview) provide a more versatile and efficient choice for multiresolution coefficients (see for instance $[19,17]$ ) than the original choice of increment coefficients [13]. MF formalisms based on the increment or wavelet coefficients anyway share a common and major drawback: they fail to compute the right part of the singularity spectrum, which is associated to scaling exponents with negative order value. Indeed isolated increments (or wavelet coefficients) can

\footnotetext{
${ }^{1}$ Note that the coefficient corresponding to $p=0$ is neces-
} sarily zero since $\zeta(0)=0$ (see Eq. (10)). be very small by chance without being located above a smooth point of the function. When raised to a negative power, this may bring a numerically predominant contribution to the structure function which is totally irrelevant. Since the cumulant exponents $c_{p}$ are defined as the Taylor expansion of $\zeta(q)$ for $q \rightarrow 0$ (cf. Section 2.2.3), they are sensitive to the $\zeta(q)$ with $q>0$ and $q<0$ and thus are poorly estimated. Moreover increment or wavelet coefficients do not provide a correct characterization of the pointwise regularity properties of data with oscillating singularities and may thus yield erroneous results even for the left part of the singularity spectrum. These drawbacks are thoroughly illustrated in Section 3.3 using the discrete wavelet coefficients ${ }^{2}$.

The WTMM methodology ${ }^{3}$ is a MF formalism, based on multi-resolution coefficients defined from the continuous wavelet transform, which has been heuristically introduced in order to compute the right part of the singularity spectrum. It has been numerically shown to compute the whole singularity spectrum of a selection of synthetic MF processes with simple singularities only. But the WTMM methodology does not receive a mathematical backing and has not been numerically assessed with synthetic MF processes with oscillating singularities.

If the signal has an oscillating singularity at $x_{0}$ of Hölder exponent $h$, then Eq. (9) is not satisfied if one chooses for $c\left(x_{0}, a\right)$ either the wavelet coefficient located as close as possible to $x_{0}$ or the wavelet coefficient on the line of maxima that points at $x_{0}$ (see [31]: these wavelet coefficients are known to decay much faster than $a^{h}$ ).

WL are multi-resolution coefficients recently introduced $[20,21]$ and numerically characterized $[22-24]$ in order to build a MF formalism which relies on exact theoretical results and is able to compute the whole singularity spectrum of functions with all kinds of singularities. The presentation and the characterization of the WL and the related MF formalism, and its application to experimental turbulent velocity data are the goals of this paper.

\subsection{Wavelet leaders}

\subsubsection{Discrete wavelet coefficients}

WL are defined from discrete wavelet coefficients (hereafter DWC). The discrete wavelet transform (see [14] for an overview) is a decomposition (also called multi-resolution analysis) of the function $f$ on the orthogonal basis $\left\{\psi_{j, k}\right\}_{j \in \mathbb{Z}, k \in \mathbb{Z}}$ composed of discrete wavelets $\psi_{j, k}$ :

$$
d(j, k)=\int_{\mathbb{R}} d x \psi_{j, k}(x) f(x)
$$

\footnotetext{
2 Wavelet transforms divide between discrete and continuous wavelet transforms and both of them provide the same characterization of pointwise regularity properties: related MF formalisms have thus equivalent validity properties [17].

3 The reader is referred to $[15,18,19,29]$ for detailed presentation and illustration of this MF formalism.
} 
with:

$$
\int_{\mathbb{R}} d x \psi_{j, k}(x) \psi_{j^{\prime}, k^{\prime}}(x)=0 \quad \text { if }(j, k) \neq\left(j^{\prime}, k^{\prime}\right)
$$

Integers $j \in \mathbb{Z}$ and $k \in \mathbb{Z}$ index the scale $a=2^{j}$ and the location $x_{0}=k 2^{j}$. Wavelets $\left\{\psi_{j, k}\right\}_{j \in \mathbb{Z}, k \in \mathbb{Z}}$ are spaceshifted and scale-dilated templates of a mother-wavelet $\psi_{0}$ :

$$
\psi_{j, k}(x)=\frac{1}{2^{j}} \psi_{0}\left(\frac{x-k 2^{j}}{2^{j}}\right)
$$

and define a basis distributed according to a dyadic basis in the space-scale plane (cf. Fig. 1). Every wavelet $\psi_{j, k}$ and then every DWC $d(j, k)$ can be associated to the dyadic interval $\lambda(j, k)$ :

$$
\lambda(j, k)=\left[2^{j} k, 2^{j}(k+1)[\right.
$$

which will be usefully used for indexing the DWC: $d(j, k)=$ $d(\lambda)$. Note that the normalization we use for the wavelet coefficients is not the standard $L^{2}$ normalization; this will be important in the the sequel in order to correctly define the WL.

\subsubsection{Definition of wavelet leaders}

WL are defined from DWC as follows:

$$
l(j, k)=\sup _{\lambda^{\prime} \subset 3 \lambda(j, k), j^{\prime} \leq j}\left|d\left(\lambda^{\prime}\right)\right|
$$

with $3 \lambda(j, k)=\lambda(j, k-1) \cup \lambda(j, k) \cup \lambda(j, k+1)$. The WL $l(j, k)$ associated to the scale $2^{j}$ and to the location $k 2^{j}$ is then the supremum of the absolute value of $\mathrm{DWC} d(j, k)$ corresponding to the same and adjacent locations $\left(k 2^{j}\right.$, $(k-1) 2^{j}$ and $\left.(k+1) 2^{j}\right)$ and to the same and smaller scales $\left(j^{\prime} \leq j\right)$ as sketched in Fig. 1 .

\subsubsection{Characterization of pointwise regularity}

If $h\left(x_{0}\right)$ is the Hölder exponent of $f$ at $x_{0}$ then $^{4}$ :

$$
\forall j, l(j, k) \sim C 2^{j h\left(x_{0}\right)},
$$

with WL (where $k$ is chosen such that $x_{0} \in \lambda(j, k)$ and $C$ is a positive constant) whereas one only has:

$$
\forall j,\left|d_{f}(j, k)\right| \sim C 2^{j h\left(x_{0}\right)}\left(1+\left|2^{-j} x_{0}-k\right|^{h\left(x_{0}\right)}\right),
$$

with DWC (note that the last characterization is a general feature of wavelet coefficients and is not specific to DWC).

WL characterize the local regularity with a local power law behavior (with exponent $h\left(x_{0}\right)$ ) whereas DWC offer a power law characterization which can be perturbed by a multiplicative term. WL thus provide a characterization of the pointwise regularity which is better suited to the derivation of a MF formalism than the one based on DWC since the derivation mainly relies on the assumption that the chosen multi-resolution coefficients do locally behave as power laws with exponent $h\left(x_{0}\right)$ (see the heuristic argument in 2.2.2).

\footnotetext{
4 The notation $a \sim b$ means that the lower limit of $\log (a) / \log (b)$ is 1 .
}

\subsection{Validity of multifractal formalisms based on the DWC and the WL}

First, note that the derivation of the MF formalism which is performed in Section 2.2.2 is correct when using the WL because of (24), whereas no similar formula exists either for the DWC or the increments. Formulas such as (24) exist only under the additional assumption that the singularity considered is a simple singularity. This explains why the WL based MF formalism has a wider range of validity.

\subsubsection{Theoretical results}

Exact results proved in [20,21] are stated in this section. Quantities $X$ will be denoted $X^{d}$ when computed with DWC and $X^{l}$ when computed with WL. $L T[X](h)$ denotes the Legendre transform of $X(q)$.

If $f$ is a uniform Hölder function ${ }^{5}$ then the WL based MF formalism satisfies the inequality ${ }^{6}$ :

$$
\forall h, \quad D(h) \leq L T\left[\zeta^{l}\right](h) .
$$

This inequality is sharper than other upper bounds that can be proved for MF formalisms based on increments or on DWC. In particular, this MF formalism has a wider range of validity.

The DWC based MF formalism also receives exact results $[17,20]$. Let us recall that $h_{0}$ is defined as the abscissa of the maximum of the singularity spectrum $D(h)$ (cf. Section 2.1.3) and define $h_{c}$ such that $q_{c} h_{c}=D\left(h_{c}\right)$ and $q_{c}=\frac{d D}{d h}\left(h_{c}\right)$, i.e., $h_{c}$ is the abscissa at which the tangent of $D(h)$ is drawn from the origin $(h, D)=(0,0)$ (cf. Fig. 2). If $f$ is a uniform Hölder function, then:

$$
\text { if } h \leq h_{c}, \quad L T\left[\zeta^{l}\right](h)=L T\left[\zeta^{d}\right](h),
$$

so that both formulas coincide in this range of exponents; furthermore if $f$ has only simple singularities:

$$
\text { if } h \leq h_{0}, \quad D(h) \leq L T\left[\zeta^{d}\right](h) .
$$

Use of this formalism can hence only provide the measurement of the left part of $D(h)$ if simple singularities

${ }^{5}$ A function $f$ is uniform Hölder of order $\alpha<1\left(f \in C^{\alpha}(\mathbb{R})\right)$ if $\exists C>0: \quad \forall x_{0}, \quad\left|f(x)-f\left(x_{0}\right)\right| \leq C\left|x-x_{0}\right|^{\alpha}$. This definition easily extends to $\alpha \geq 1$.

${ }^{6}$ Note that the maximum of $L T\left[\zeta^{l}\right](h)$ is set to $L T\left[\zeta^{l}\right]\left(h_{0}\right)=$ 1 as a result of Def. (10). Displayed definitions are thus suited to the MF analysis of functions with singularity spectra reaching their maximum at value $D\left(h_{0}\right)=1$ only (meaning that the analyzed function has singularities spread over a spacefilling set). The MF formalism based on the WL could be easily adapted to the general case where $D\left(h_{0}\right) \leq 1$ [21], whereas by definition the WTMM methodology is able to perform the MF analysis of functions with $D\left(h_{0}\right) \leq 1$. Functions with $D\left(h_{0}\right)=1$ are anyhow the only ones considered in this present contribution (as previous works [32, 28,33] have shown that turbulent Eulerian velocity data can be described by such functions). 
only are present, and even of a smaller part of it if oscillating singularities are present. In other words the right part of $D(h)$ cannot be estimated with DWC and the computation of the left part itself can even fail as a result of the presence of oscillating singularities.

The formalism based on the WL then brings a real quantitative enhancement: It yields a comprehensive MF analysis, i.e., the estimation of the whole singularity spectrum $D(h)(\forall h)$ regardless of whether simple singularities are the only ones present or not. These results are sketched in Fig. 2. In addition to that, we point out that it relies on mathematically well-fonded basis, in contrast to the WTMM methodology for which no exact result exists (though its validity has been numerically checked with synthetic MF processes with simple singularities). Eventually note that another advantage of the WL is that one can show that scaling exponents are independent of the (smooth) wavelet which is used; a property which does not hold for the DWC, and is an open issue for the WTMM.

The WL based MF formalism is valid when the inequality (26) becomes an equality, which implies in turn a concave $^{7}$ singularity spectrum since a Legendre transform of any function is necessarily a concave function. Even though there exists some counter-examples, most mathematical models used in physics (for instance all models used in turbulence [4]) or defining synthetic MF data result in concave singularity spectra and satisfy the WL based MF formalism. The following usual assumption is thus reliably made: only uniform Hölder functions with concave singularity spectrum will be considered in the sequel and results obtained on real data will be interpreted as equalities and not inequalities. Note that this assumption implies that the DWC based MF formalism is valid for $h \leq h_{c}$ or $h \leq h_{0}$ if simple singularities only are present.

\subsubsection{Illustration \#1: oscillating singularities}

WL always characterize the local regularity with a local power law behavior (with exponent $h\left(x_{0}\right)$ ) whereas DWC offer a power law characterization which can be perturbed by a multiplicative term (cf. Eqs. (24) and (25)). This difference is illustrated in Fig. 3 where $d(j, k)$ and $l(j, k)$ are computed for an isolated simple singularity (with $h=$ 0.6 ) and an isolated oscillating singularity (with $(h, \beta)=$ $(0.6,1))$. WL exhibit a power law behavior with the correct exponent both for the cusp and the chirp whereas DWC behave like a power law (with the correct exponent) only for the cusp. Since the validity of a MF formalism mainly relies on the assumption that multi-resolution coefficients do locally behave as power laws with exponent $h\left(x_{0}\right)$ (cf. Section 3.2.3), this simple example clearly points out at the failure of the DWC based MF formalism ${ }^{8}$ to correctly compute the singularity spectrum of functions with oscillating singularities when $h_{c} \leq h \leq h_{0}$.

\footnotetext{
7 A function is concave if $\forall x, y$ with $t \in[0,1]$, then $f(t x+(1-t) y) \geq t f(x)+(1-t) f(y)$.

8 This is actually a general result valid for all wavelet coefficients, either discrete or continuous, and also for increment coefficients.
}

\subsubsection{Illustration \#2: scaling exponents with negative orders and the right part of the singularity spectrum}

The right part of $D(h)$ (cf. Section 2.1.3) is related to scaling exponents $\zeta(q)$ with negative order $(q<0)$. The $\zeta(q)$ are defined from the structure functions $S(q, a)$ which are estimates (by space-averaging) of the $q$-th order statistical moments of the $c\left(x_{0}, a\right)$ (cf. Eq. (10)). A meaningful estimation of the statistical moments of the $c\left(x_{0}, a\right)$ with a negative order implies that at least the chosen $c\left(x_{0}, a\right)$ have a probability distribution function which is zero-valued for $c\left(x_{0}, a\right)=0$.

The random wavelet cascade process is a synthetic MF process commonly used to benchmark MF formalisms (see for instance [22-24]) and model turbulent velocity (see [34] for a thorough definition) and which MF properties can be easily prescribed. Histograms of the absolute value of DWC and WL computed on a realization of the process are plotted in Fig. 4 and show that the computation of statistical moments of the $d(j, k)$ with negative order is meaningless in contrast of those of the $l(j, k)$. This clearly illustrates that the DWC based MF formalism fails to provide the right part of the singularity spectrum since it is not able to compute scaling exponents with negative order.

\subsection{Detection of oscillating singularities}

In spite of various attempts [9-12] the detection of oscillating singularities remains an open issue in signal processing. This section points out that the failure of the DWC based MF formalism when performing a MF analysis of data with oscillating singularities can be actually exploited. Indeed, since the WL based MF formalism is expected to be valid in the same situation, a discrepancy between the results given by these two formalisms in the range $\left[h_{c}, h_{0}\right]$ is a signature of the presence of oscillating singularities.

In turn it is worth noting that an agreement of the results between the two MF formalisms should not be taken as a proof of the absence of oscillating singularities. The two formalisms might indeed give coinciding results if oscillating singularities exist but bring a minor contribution to the singularity spectrum. Let us define $D(h, \beta)$ (called the grand-canonical spectrum $[11,12]$ ) as the Hausdorff dimension of the subset of points $x_{0}$ at which the Hölder and oscillating exponents take the specific values $h$ and $\beta$ (see Eq. $(5))^{9}$. The singularity spectrum $D(h)$ then straightforwardly relates to $D(h, \beta)$ :

$$
D(h)=\sup _{\beta} D(h, \beta) .
$$

If $D(h)=D(h, \beta)$ with $\beta=0$, oscillating singularities bring a minor contribution to $D(h)$ since the simple singularities $(\beta=0)$ with Hölder exponent value $h$ are spatially distributed on a subset with a larger Hausdorff dimension

\footnotetext{
9 A proper definition of the oscillating exponent $\beta$ exists [31] but is not discussed here.
} 
than the ones characterizing the oscillating singularities $(\beta \neq 0)$ with the same Hölder exponent value.

The exact behavior of the DWC based MF formalism for data with oscillating singularities that bring a minor contribution to $D(h)$ is still not fully understood [35] and is under current investigation (see however $[32,36]$ for some preliminary results in this direction). This topic is then not further discussed in this paper and the following partial but cautious conclusion is drawn: if an agreement between the Legendre transforms of the $\zeta^{d}(q)$ of the $\zeta^{l}(q)$ is observed for $h_{c} \leq h \leq h_{0}$, there is either no oscillating singularities or oscillating singularities that bring a minor contribution to the singularity spectrum $D(h)$. In turns a discrepancy between the results of the two MF formalisms in that range proves the presence of oscillating singularities.

\section{Multifractality and turbulence}

\subsection{Multifractal models}

Several MF models have been proposed for the turbulent Eulerian velocity (see [4] for an overview). The two most commonly used are the log-normal model (after the work by Obukhov and Kolmogorov [2,3]) and the She-Lévêque model [37] which both relate to a multiplicative cascade process down the scales (both models can be seen as two specific cases of the more general framework of the infinitely divisible cascades $[8,38,39])$. The MF properties of these processes are controlled by the statistics of the multipliers: the log-normal model corresponds to log-normal statistics whereas the She-Lévêque model corresponds to log-Poisson statistics [40]. It is noted that both models predict MF functions with simple singularities only.

The log-normal model predicts quadratic expressions for both the singularity spectrum and the scaling exponents:

$$
\begin{gathered}
D_{l n}(h)=\left\{\begin{array}{ll}
1+\frac{\left(h-c_{1}\right)^{2}}{c_{2}} & \text { if } h_{*}^{+} \leq h \leq h_{*}^{-} \\
\text {else }
\end{array},\right. \\
\zeta_{l n}(q)= \begin{cases}c_{1} q+\frac{c_{2}}{2} q^{2}, & q_{*}^{-} \leq q \leq q_{*}^{+} \\
1+q h_{*}^{-}, & q \geq q_{*}^{+} \\
1+q h_{*}^{+}, & q \leq q_{*}^{-}\end{cases}
\end{gathered}
$$

with $c_{1}=1 / 3-3 c_{2} / 2$ (so that $\zeta_{\ln }(3)=1$ ) while the SheLévêque model predicts:

$$
\begin{gathered}
D_{S L}(h)= \begin{cases}\left(h-\frac{1}{9}\right)\left[A_{1}-A_{2} \ln \left(h-\frac{1}{9}\right)\right]-2 \\
\text { if } h_{*}^{+} \leq h \leq h_{*}^{-} \\
-\infty\end{cases} \\
\zeta_{S L}(q)= \begin{cases}\frac{q}{9}+2\left[1-\left(\frac{2}{3}\right)^{q / 3}\right], & q_{*}^{-} \leq q \leq q_{*}^{+} \\
1+q h_{*}^{-}, & q \geq q_{*}^{+} \\
1+q h_{*}^{+}, & q \leq q_{*}^{-}\end{cases}
\end{gathered}
$$

with $A_{1}=3\left(\frac{1+\ln \left(\ln \left(\frac{3}{2}\right)\right)}{\ln \left(\frac{3}{2}\right)}-1\right), A_{2}=\frac{3}{\ln \left(\frac{3}{2}\right)}$. (The reader can refer to $[41,22,42]$ for a thorough discussion of the linear behavior of the scaling exponents beyond the critical orders $q_{*}^{+}$and $q_{*}^{-}$.) Critical parameters $h_{*}^{+}, h_{*}^{-}, q_{*}^{+}$and $q_{*}^{-}$ are for the log-normal model:

$$
\begin{gathered}
h_{*}^{+}=c_{1}-\sqrt{-2 c_{2}}, \quad h_{*}^{-}=c_{1}+\sqrt{-2 c_{2}}, \\
q_{*}^{+}=-q_{*}^{-}=\sqrt{-2 / c_{2}}
\end{gathered}
$$

and for the She-Lévêque model:

$$
\begin{aligned}
& h_{*}^{+} \simeq 0.162, \quad h_{*}^{-} \simeq 0.694, \\
& q_{*}^{-} \simeq-5.69, \quad q_{*}^{+} \simeq 12.36 .
\end{aligned}
$$

Eqs. (29), (30), (31) and (32) are predictions for 1D turbulent Eulerian velocity, i.e., 1D geometrical cuts of $3 \mathrm{D}$ velocity fields. Experimental velocity data (cf. section 5.1) are indeed measurements of the streamwise component of $1 \mathrm{D}$ geometrical cuts of the original 3D turbulent field, and experimental MF results should thus be compared to last equations.

The log-normal model has one free parameter, the intermittency coefficient $c_{2}$ (which coincides with the second order cumulant exponent), which commonly accepted value is $c_{2}=-0.025[32,43,44,28]$ (the She-Lévêque model has no free parameter). Both models are MF models (as opposed to monofractal ones) and the corresponding cumulant exponents are listed in Table 2 . The scaling exponents and the singularity spectra predicted by these models are plotted in Fig. 6 and Fig. 7. It is noted that predictions for the left part of the singularity spectrum (or for scaling exponents with positive order) are numerically very close whereas they clearly differ for the right part of $D(h)$ (or for $\zeta(q)$ with $q<0$ ). This remark illustrates the importance of designing tools allowing a complete MF analysis, i.e., the computation of both the left and the right part of the singularity spectrum.

\subsection{Multifractality and universality}

Let us discuss an important assumption of the MF description of turbulence. A turbulence experiment is necessarily characterized by a finite Reynolds number $R_{\lambda}{ }^{10}$ and the large fluctuations observed at inertial scales are smoothed out at smallest scales by viscous forces (which become predominant at the Kolmogorov scale): velocity spatial profiles are not locally singular (they possess at least a first order derivative at every point). The MF description (and thus the associated singularity spectrum models) is actually related with the pointwise regularity properties of the turbulent velocity in the limit of infinite Reynolds numbers: $R_{\lambda} \rightarrow+\infty$. In other words it is assumed that the scaling properties observed at inertial scales extend down

\footnotetext{
10 The Taylor scale based Reynolds number, called Reynolds number in the sequel, is defined as: $R_{\lambda}=\frac{\sigma \lambda}{\nu}$ where $\sigma$ is the standard deviation of the velocity $v(x), \lambda=\sqrt{\frac{\sigma^{2}}{\mathbb{E}\left(\frac{\partial v}{\partial x}\right)^{2}}}$ is the so-called Taylor scale and $\nu$ the kinematic viscosity.
} 
to all smallest scales, i.e., $a \rightarrow 0$, and thus that velocity has the pointwise regularity properties characterized by the singularity spectrum $D(h)$ associated to these scaling properties. This assumption is entirely consistent since the same mechanism (a multiplicative cascade through the scales) is used to model both the scaling properties of structure functions and the (asymptotic) pointwise regularity properties.

All theoretical predictions about the singularity spectrum of turbulent velocity thus implicitly concern the limit $R_{\lambda} \rightarrow+\infty$. This is a universal description of turbulence since statistical properties of any turbulent flow (turbulent jet, grid turbulence, ...) are expected to coincide in that limit: all turbulent flows are hence characterized by common scaling exponents $\zeta(q)$ and singularity spectrum $D(h)$. The value of $\zeta(3)$ is for instance predicted by the Kolmogorov's four fifths law [26] (derived from the KármánHowarth equation [45]) which is an exact result for any turbulent flow in the limit of $R_{\lambda} \rightarrow+\infty$ : the third order structure function is an exact power law with $\zeta(3)=1$.

It is known that the scaling exponent $\zeta(3)$ computed from experimental data departs from 1 and does not take the same value for all experiments, which is also true for any other value of the order $q$. It is known as well (see for instance [46]) that the normalized scaling exponents:

$$
\tilde{\zeta}(q)=\frac{\zeta(q)}{\zeta(3)}
$$

(which necessarily take the specific value: $\tilde{\zeta}(3)=1$ ) do collapse on a common curve for different experiments, supporting the fact that the normalized scaling exponents $\tilde{\zeta}(q)$ (and thus their Legendre transform $\tilde{D}(h)$ ) are universal in contrast to the raw scaling exponents $\zeta(q)$ (this will be illustrated in Section 5.4.1). Results about universal scaling and/or MF properties of turbulence are hence usually discussed using the $\tilde{\zeta}(q)$ and not the $\zeta(q)$. Note that the coefficients of the Taylor series expansion of $\tilde{\zeta}(q)$ around $q=0$ will be called normalized cumulant exponents and denoted by $\tilde{c}_{p}$.

The collapse of the $\tilde{\zeta}(q)$ computed from different experiments implies that the non-universal component of the $\zeta(q)$, i.e., due to the specific nature of the experimental device (turbulent jet, grid turbulence, ... but also the specific value of the necessarily finite Reynolds number), can be taken into account with a multiplicative parameter $C\left(R_{\lambda}, \ldots\right)$ only:

$$
\zeta(q)=C\left(R_{\lambda}, \ldots\right) \tilde{\zeta}(q) .
$$

The value of $C\left(R_{\lambda}, \ldots\right)$ is experimentally given by the computation of $\zeta(3)$ but also receives quantitative expression for specific cases of turbulent flows [22] which are derived from a finer approximation (which includes finite Reynolds number effects) of the Kármán-Howarth equation (report as well to [47-51] which are recent works about the discrepancy between experimental the third order structure function and the four fifths law; see as well [52]). Additional considerations on this topic will be discussed in a forthcoming article.

\subsection{Goal of multifractal analysis in turbulence}

MF analysis of turbulent velocity data aims at answering several questions. The first of those is the investigation of the dependence of the MF properties with the Reynolds number and the assessment of the universal MF description relevance. The second is the issue of the possible presence of oscillating singularities in turbulent velocity data. The last question is the discrimination between proposed MF models.

All these important issues require the use of a comprehensive MF formalism (computation of the whole singularity spectrum for functions with all kinds of singularities) to receive clear and reliable answers.

\section{Application to Fully Developped Turbulence Velocity}

\subsection{Experimental data and analysis details}

Several sets of turbulent Eulerian velocity data are analyzed in this paper. They all consist of hot wire probe measurements of the velocity streamwise component. Their characteristics are summarized in Table 1. The first data set concerns the experiment performed by Chanal et al. [43]. The same experimental device has been used to generate cold helium jet with different values of the Reynolds number: $R_{\lambda} \simeq 90,210,460$ and 930 . Note that these four data sets are characterized by the same integral scale. The two other data sets have been performed at the ONERA Modane wind tunnel and their Reynolds numbers are: $R_{\lambda} \simeq 2500$ (1986 campaign) and $R_{\lambda} \simeq 2000$ (1995 campaign). The two previous data sets have been made available to us by Y. Gagne (Laboratoire des Écoulements Géophysiques et Industriels, Université Joseph Fourier, INPG and CNRS, Grenoble, France).

The Taylor hypothesis of frozen turbulence (see, e.g., [53]) for the Modane wind tunnel data or its local version for the helium jet is used in order to convert raw temporal data into $1 \mathrm{D}$ spatial profiles of the velocity streamwise component $^{11}$.

All data are split into series of almost 32 integral scales duration (cf. Table 1) in order to estimate the confidence intervals of the computed quantities (the confidence interval used all along this paper is the common $95 \%$ confidence interval for the empirical average estimator on $N$ samples of a Gaussian random variable: $\pm 2 \frac{\sigma}{\sqrt{N}}$ where $\sigma$ is the estimated standard deviation) and also to check the statistical convergence of all results displayed in this contribution (for instance the cumulant exponents up to order 4).

11 The Taylor hypothesis is valid if the standard deviation of the velocity is sufficiently small compared to its mean. This last condition is fulfilled for the Modane wind tunnel data (cf. Table 1) whereas a local version of the Taylor hypothesis is required for the helium jet data. The corresponding data processing has been performed by Chanal et al. [43] who provided us with spatial profile data. 
Eventually all analyses presented are obtained using the Daubechies wavelet [54] with 3 vanishing moments (the regularity of this wavelet ${ }^{12}$ is larger than the largest Hölder exponent $h$ present in data under analysis). Robustness with respect to variations of the number of vanishing moments is known to be a key issue for scaling analysis (cf. [55]). Other discrete wavelets (with regularity larger than the largest Hölder exponent) have thus been used but no discrepancy in the results have been observed: displayed results are thus independent of the used discrete wavelet basis.

\subsection{Effect of the length of the inertial scale range}

The first step in the use of any MF formalism is to check that computed structure functions $S(q, a)$ indeed behave like power laws. Structure functions of turbulent velocity data, computed with wavelet or increment coefficients, are known to have such a behavior within the so-called inertial range, that roughly speaking extends from the integral scale $L_{i}$ down to the Taylor scale $\lambda$. The value of the integral scale is controlled by the physical processes with which the energy is injected in the turbulent flow whereas the inertial range is limited at smallest scales by dissipative effects: the viscosity becomes predominantly effective and structure functions do not behave like power laws anymore. The extend of the inertial range is mainly controlled by the Reynolds number $R_{\lambda}$ and increases with it (the ratio of $L_{i}$ to $\lambda$ is proportional to $R_{\lambda}$ according to dimensional analysis).

DWC correctly characterize MF properties of turbulent velocity within the inertial range: the $S^{d}(q, a)$ (with $q>0$ ) do behave like power laws in this range as a result. Linear regressions are performed within a carefully selected scale range $\left[a_{\min }=2^{j_{\min }}, a_{\max }=2^{j_{\max }}\right]$ in order to compute the scaling exponents $\zeta^{d}(q)$, where $a_{\min }$ and $a_{\max }$ (which are close to $\lambda$ and $L_{i}$, respectively) delimit the observed power laws.

The situation is actually different when using the WL. By definition the coefficient $l(j, k)$ accounts for the local properties around time $2^{j} k$, at scale $2^{j}$ but also at smaller scales: $2^{j^{\prime}}$ with $j^{\prime}<j$. Hence the $l(j, k)$ can correctly characterize the MF properties only if the $d\left(j^{\prime}, k\right)$ account for these properties at scale $2^{j}$ but also at smaller scale $2^{j^{\prime}}$. In turn, if a power law is observed for the $S^{d}(q, j)$ starting at a minimal scale $2^{j_{\text {min }}}$, the $S^{l}(q, j)$ are expected to behave like power laws only starting at a larger scale $2^{j_{\min }+m}$ with $m \geq 1$. As a consequence the range of scales which can be used to compute the $\zeta^{l}(q)$ is shorter than the one used to compute the $\zeta^{d}(q)$.

This is illustrated in Fig. 5 which shows third order structure functions computed with DWC $\left(S^{d}(3, j)\right)$ and WL $\left(S^{l}(3, j)\right)$ from different velocity data sets performed with the same experimental device but characterized by four different Reynolds number: $R_{\lambda} \simeq 90,210,460$ and

\footnotetext{
12 The regularity of a wavelet $\psi$ is the smallest value of $\alpha$ such that $\psi \in C^{\alpha}(\mathbb{R})$ (see Section 3.2.3).
}

930 (cf. Section 5.1). The inertial scale range extends toward smallest scales when $R_{\lambda}$ increases ${ }^{13}$. The local exponent is defined at octave $j$ as:

$(S(3, j+1 / 2)-S(3, j-1 / 2))$ : if $S(3, j)$ is a pure power law with exponent $\zeta(3)$ then the local exponent is constant and coincides with $\zeta(3)$, and if $S(3, j)$ behaves like a power-law over a given range the local exponent is almost constant within that range. No power law behavior is observed for $S^{d}(3, j)$ with $R_{\lambda} \simeq 90$ or $R_{\lambda} \simeq 210$ but with $R_{\lambda} \simeq 460$ and $R_{\lambda} \simeq 930$ : the local exponent is almost constant over a sufficiently large range to perform a reliable scaling exponent computation. In contrast, $S^{l}(3, j)$ only clearly behaves like a power law for the highest $R_{\lambda}$ value $\left(R_{\lambda} \simeq 930\right)$. As a consequence $\zeta^{d}(3)$ can be reliably estimated for $R_{\lambda} \simeq 460$ but not $\zeta^{l}(3)$ : a power law behavior is observed over almost 3 octaves for $S^{d}(3, j)$ but only over 1 octave for $S^{l}(3, j)$, thus preventing any reliable measurement. According to the heuristic discussion developed in the previous paragraph, it means that WL at scale $2^{j}$ are mainly sensitive to DWC at scales $2^{j}, 2^{j-1}$ and $2^{j-2}$ and that almost two less octaves are available for the scaling exponent computation.

This discussion illustrates one important point: if the Reynolds number is moderately large (e.g., $R_{\lambda} \simeq 460$ for this specific experiment), one can compute the left part only (or even the part defined by $h<h_{c}$ ) of the singularity spectrum by using the DWC but not its right part since the computation of the $\zeta^{l}(q)$ cannot be performed. Note that the WTMM methodology suffers from the same drawback: power laws are established with the same "scale delay" [22].

\subsection{Computation of scaling exponents and cumulant exponents}

The remaining of this article will focus on data sets for which the scaling exponents $\zeta^{l}(q)$ and thus the WL based MF formalism can be reliably used, i.e., the velocity data sets corresponding to $R_{\lambda} \simeq 930,2000$ and 2500 (cf. Section 5.1). Raw and normalized scaling exponents, cumulant exponents and singularity spectra are computed using the WL and the DWC. Scale ranges over which linear regressions are performed are carefully chosen according to the discussion made in Section 5.2. Eventually note that the large durations of analyzed data (cf. Table 1) ensure the statistical convergence of all displayed results.

Computed $\zeta^{l}(q)$ and $\tilde{\zeta}^{l}(q)$ and their Legendre transforms are displayed in Figs. 6, 7, 8 and 9. The cumulants $C^{l}(p, a)$ computed on the data set with $R_{\lambda} \simeq 2000$ are plotted in Fig. 10 (similar plots are obtained with other data sets). Estimated values of cumulant exponents $c_{p}$ and $\tilde{c}_{p}$ are listed in Tables 3 and 4.

\footnotetext{
13 Note that the integral scale $L_{i}$ of all these data sets is the same since being fixed by the experimental device.
} 


\subsection{Discussions}

\subsubsection{Dependence of the singularity spectrum on the Reynolds number}

The dependence of MF properties with the Reynolds number $R_{\lambda}$ is now discussed from Figs. 6 and 7. A dependence with the Reynolds number is observed: the $\zeta^{l}(q)$ computed for the three Reynolds numbers $R_{\lambda} \simeq 930,2000$ and 2500 do not coincide (note in particular that $\zeta^{l}(3)$ is always smaller than 1 but gets closer to 1 when $R_{\lambda}$ increases as shown in Table 3). As a direct consequence their Legendre transforms $D^{l}(h)$ do not coincide and cumulant exponents $c_{p}^{l}$ are distinct (cf. Table 3 ). In contrast normalized scaling exponents $\tilde{\zeta}^{l}(q)$ very clearly collapse on a common curve for the full range of computed orders $q$, as do their Legendre transforms. Normalized cumulant exponents $\tilde{c}_{p}^{l}$ are in a very good agreement for all data sets (cf. Table 4).

MF properties of turbulent velocity depend on specific details of the experimental flow (for instance the Reynolds number) and are thus not universal. MF properties of experimental data can anyway be related to those of turbulent flows with $R_{\lambda} \rightarrow+\infty$, which are universal, through the commonly used normalized scaling exponents as discussed in Section 4.2.

\subsubsection{Investigation of the existence of oscillating singularities}

The methodology proposed in Section 3.4 is used to assess the existence of oscillating singularities in experimental turbulent velocity data. The Legendre transform of scaling exponents computed with the DWC $\left(D^{d}(h)\right)$ is compared to the Legendre transform of scaling exponents computed with the WL $\left(D^{l}(h)\right)$, in particular for $h$ values close to the abscissa of the observed maxima (located at $h_{0} \simeq 0.34$ ) for the data set corresponding to $R_{\lambda} \simeq 2000$ (see Fig. $9)$. $D^{d}(h)$ and $D^{l}(h)$ clearly coincide on the whole left part of the singularity spectrum: $h \leq h_{0}$. Same results are obtained with data sets characterized by $R_{\lambda} \simeq 930$ and $R_{\lambda} \simeq 2500$.

The conclusion is that no oscillating singularity is detected in 1D turbulent Eulerian velocity data with the proposed methodology. According to the discussion made in Section 3.3.2 one cannot conclude to the absence of oscillating singularities but if such singularities exist, their contribution to MF properties is minor.

Two possible interpretations can be drawn from this result. The first is that no oscillating singularity is to be found within turbulent Eulerian velocity fields. This backs the use of MF models based on multiplicative cascades (such as the log-normal or log-Poisson discussed above) since they result in MF functions with simple singularities only. The second is that oscillating singularities may exist in 3D turbulent velocity fields but not in 1D turbulent velocity data, which are 1D geometrical cuts of actual $3 \mathrm{D}$ fields. Indeed, if singularities with Hölder exponent $h$ are present on a set with dimension $\delta_{h}$ in the $3 \mathrm{D}$ field, they can be recovered from $1 \mathrm{D}$ geometrical cuts if $\delta_{h}>2$ only, appearing in that case as singularities with Hölder exponent $h$ on a set of dimension $\delta_{h}-2$ (see [56] for theoretical arguments backing this heuristic argument). On the other hand these singularities will be absent from 1D geometrical cuts if $\delta_{h}<2$. It is worth noting that if oscillating singularities are assumed to be the signatures of coherent vortices of the turbulent flow, they would be associated with $\delta_{h}=1$ and be absent in 1D geometrical cuts. A definitive answer can thus be derived only from the complete MF analysis of 3D turbulent velocity data (from DNS for instance). As discussed in Section 5.2 the use of WL requires data with sufficiently high Reynolds number and we do not have access to such data thus far.

Several works [9-12] had addressed the issue of the existence of oscillating singularities in experimental data. This is the first time that this issue receives an answer, though partial, from data analysis. This has been achieved because the WL allow to define a MF formalism valid for all kinds of singularities since they better characterize the local regularity properties (see Section 3.2.3).

\subsubsection{Discrimination between multifractal models}

This section discusses the universal MF properties of turbulent velocity data, focusing on the right part of the normalized singularity spectrum $\tilde{D}(h)$ and on the values of the normalized cumulant exponents $\tilde{c}_{p}$. As discussed in the Section 4.1 the computation of the right part of $\tilde{D}(h)$ $\left(h \geq h_{0}\right)$ is essential for the discrimination between the log-normal and She-Lévêque models. Figs. 6 and 7 shows that all computed singularity spectra are in very good agreement with the log-normal model and clearly depart from the She-Lévêque model, within confidence intervals (see Fig. 8 for $R_{\lambda} \simeq 2000$; similar results are obtained for $R_{\lambda} \simeq 930$ and 2500). Note that the log-normal model has one free parameter $\left(c_{2}\right)$ which has been chosen in Figs. 6 and 7 as $c_{2}=-0.025$, which is its commonly accepted value $[32,43,44,28]$ and not adjusted on order to fit the computed $\tilde{D}^{l}(h)$.

Normalized cumulant exponents $\tilde{c}_{p}^{l}$ are computed up to order $p=4$ (cf. Table 4 ) and are found to take common values (within confidence intervals):

$$
\left\{\begin{array}{lr}
\tilde{c}_{1}^{l} \simeq & 0.372 \pm 0.004 \\
\tilde{c}_{2}^{l} \simeq & -0.025 \pm 0.002 \\
\tilde{c}_{3}^{l} \simeq 0.000 \pm 0.001 \\
\tilde{c}_{4}^{l} \simeq 0.000 \pm 0.001
\end{array}\right.
$$

These values exhibit a clear agreement with the log-normal model since only $\tilde{c}_{1}^{l}$ and $\tilde{c}_{2}^{l}$ are found to have non zero values. The computed values of $\tilde{c}_{1}^{l}$ and $\tilde{c}_{2}^{l}$ furthermore satisfy the condition $\tilde{c}_{1}^{l}=1 / 3+3 / 2 \tilde{c}_{2}^{l}$ imposed by the log-normal model (cf. Section 4.1) and the computed value of $\tilde{c}_{2}^{l}$ coincides with previous estimations $[32,43,44,28]$. Note that the log-normal model has been sometimes rejected arguing a violation of the Novikov's condition [57]. This wrong conclusion has been drawn assuming a quadratic behavior of the scaling exponents for all orders $q$, whereas the $\zeta(q)$ necessarily behave like a linear function of $q$ beyond the 
critical orders $q_{*}^{+}$and $q_{*}^{-}$(cf. Eq. (30)): the log-normal model (with $c_{2}=-0.025$ ) actually satisfies the Novikov's condition (see $[41,22,42]$ for a thorough discussion of the asymptotic linear behavior of scaling exponents).

These results confirm those previously obtained using the WTMM methodology [32,28,33]: observed universal MF properties are compatible with a log-normal model for the singularity spectrum of turbulent Eulerian velocity and are fully characterized by the value of the second order cumulant exponent: $\tilde{c}_{2} \simeq-0.025 \pm 0.002$. Note finally that these conclusions are drawn directly from the computation of scaling exponents (and their Legendre transforms). Therefore they do not use the hypothesis that the MF formalism holds. Our discrimination results remain valid even if it is not the case.

\section{Conclusions}

The MF description is one of the routes that lead toward a statistical description of turbulence within the inertial scales. It ties the scaling properties of turbulence to its local regularity properties (Section 2) and also relates the observed MF properties to the underlying multiplicative cascade structure of turbulence (Section 4). The estimation of turbulence MF properties from experimental data thus provides meaningful inferences about physical processes which originate turbulence. A comprehensive MF analysis of experimental data which relies on well-founded mathematical basis should hence be performed in order to unequivocally establish the MF properties of turbulent velocity and thus safely extract information about physical processes involved in turbulent flows.

The WL based MF formalism [20,21], thoroughly described and illustrated in this article (Section 3), addresses successfully the three following requests: (1) exact computation of the whole singularity spectrum; (2) mathematically well-founded basis; and (3) validity for all kinds of singularities. The WL based MF formalism is applied for the first time to experimental turbulent velocity data (Section 5). Methodological aspects are first thoroughly discussed and it is shown that only velocity data with sufficiently large $R_{\lambda}$ can be analyzed. The MF analysis of three data sets from different experimental devices and with different $R_{\lambda}$, thus yielding strong inferences, is then performed. The dependence of MF properties on the specific value of $R_{\lambda}$ is carefully described and the collapse of normalized quantities $\left(\tilde{\zeta}(q), \tilde{D}(h)\right.$ and $\left.\tilde{c}_{p}\right)$ characterizing the universal part of MF properties is discussed. The issue of the existence of oscillating singularities receives for the first time a clear though partial answer: no oscillating singularities are detected, which means that oscillating singularities might exist but only if bringing a minor contribution to MF properties along the discussion made in Section 3.4. A complete and definitive answer is still to be brought to this important question and the WL might be the first step toward this achievement. Eventually the universal MF properties of turbulent velocity are compared to those of proposed MF models and are accurately parameterized with cumulant exponents up to order 4 . The log-normal model is clearly shown to correctly account for all observed universal MF properties and its only free parameter is estimated: $\tilde{c}_{2}=-0.025 \pm 0.002$. These results confirms those previously obtained in $[28,32,43,44$, $33]$. The contribution of this article toward the issue of discriminating between various MF models is anyway of first importance since a complete MF analysis is done using mathematically well-founded tools. 


\section{References}

1. A.N. Kolmogorov, Dokl. Akad. Nauk SSSR 30, 299 (1941)

2. A. Obukhov, J. Fluid Mech. 13, 77 (1962)

3. A.N. Kolmogorov, J. Fluid Mech. 13, 82 (1962)

4. U. Frisch, Turbulence, the Legacy of A.N. Kolmogorov (Cambridge University Press, 1995)

5. A. Yaglom, Dokl. Akad. Nauk. SSR 166, 49 (1966)

6. B. Mandelbrot, J. Fluid. Mech. 62, 331 (1974)

7. L. Richardson, Weather prediction by numerical process (Cambridge University Press, 1922)

8. B. Castaing, Y. Gagne, E. Hopfinger, Physica D 46, 177 (1990)

9. J. Hunt, J. Vassilicos, Proc. R. Soc. Lond. A 434, 183 (1991)

10. N.R. Kevlahan, J. Vassilicos, Proc. R. Soc. Lond. A 447, 341 (1994)

11. A. Arneodo, E. Bacry, J. Muzy, Phys. Rev. Letters 74, $4823(1995)$

12. A. Arneodo, E. Bacry, S. Jaffard, J. Muzy, J. Stat. Phys. 87(1-2), 179 (1997)

13. G. Parisi, U. Frisch, On the singularity structure of fully developed turbulence, appendix to Fully developed turbulence and intermittency by U. Frisch, in Proc. Int. Summer school Phys. Enrico Fermi, North Holland (1985)

14. S. Mallat, A Wavelet Tour of Signal Processing (Academic Press, San Diego, CA, 1998)

15. J. Muzy, E. Bacry, A. Arneodo, Phys. Rev Lett. 67, 3515 (1991)

16. C. Meneveau, J. Fluid Mech. 232, 469 (1991)

17. S. Jaffard, S.I.A.M. J. Math. Anal. 28(4), 944 (1997)

18. J. Muzy, E. Bacry, A. Arneodo, Phys. Rev. E 47, 875 (1993)

19. A. Arneodo, E. Bacry, J. Muzy, Physica A 213, 232 (1995)

20. S. Jaffard, Wavelet Techniques in Multifractal Analysis, in "Fractal Geometry and Applications: A Jubilee of Benoit Mandelbrot", ed.: M. Lapidus and M. van Frankenhuysen, Proc. of Symp. in Pure Mathematics (AMS, 2004)

21. S. Jaffard, Ann. Fac. Scien. Toul. 15(1), 3 (2006)

22. B. Lashermes, Ph.D. thesis, École Normale Supérieure de Lyon (2005)

23. B. Lashermes, S. Jaffard, P. Abry, Wavelet Leaders Based Multifractal Analysis, in ICASSP 2005 Conference, Philadelphia, USA (2005)

24. S. Jaffard, B. Lashermes, P. Abry, Wavelet Analysis and Applications, T. Quian et al eds, in Applied and Numerical Harmonic Analysis (Springer, 2006)

25. M. Schroeder, Fractals, Chaos, Power Laws. Minutes from an Infinite Paradise (W.H. Freeman and Company, 1991)

26. A.N. Kolmogorov, Dokl. Akad. Nauk SSSR 32, 16 (1941)

27. A. Arneodo, S. Manneville, J. Muzy, Eur. Phys. J. B 1, $129(1998)$

28. J. Delour, J. Muzy, A. Arneodo, Eur. Phys. J. B 23, 243 (2001)

29. V. Venugopal, S. Roux, E. Foufoula-Georgiou, A. Arneodo, Water Resour. Res. 42(6), W06D14 (2006)

30. B. Lashermes, E. Foufoula-Georgiou, submitted to Water Resour. Res. (2007)

31. S. Jaffard, Y. Meyer, Memoirs of the AMS. 123(587), 1 (1996)

32. A. Arneodo, E. Bacry, S. Jaffard, J. Muzy, J. Four. Anal. Appl. 4(2), 159 (1998)

33. S. Roux, Ph.D. thesis, Université d'Aix-Marseille II (1996)
34. A. Arneodo, E. Bacry, J. Muzy, J. Math. Phys. 39(8), 4142 (1998)

35. S. Jaffard, J. Math. Phys. 39, 4129 (1998)

36. C. Melot, J. Math. Pures Appl. 83(9), 367 (2004)

37. Z.S. She, E. Lévêque, Phys. Rev. Letters 72, 336 (1994)

38. B. Castaing, B. Dubrulle, J. Phys. II France 5, 895 (1995)

39. E. Novikov, Phys. Rev. E 50, R3303 (1994)

40. B. Dubrulle, Phys. Rev. Lett. 73, 959 (1994)

41. B. Lashermes, P. Abry, P. Chainais, Int. J. of Wavelets, Multiresolution and Information Processing 2(4), 497 (2004)

42. B. Lashermes, C. Baudet, P. Abry, P. Chainais, Limitation of Scaling Exponent Estimation in Turbulence, in Advances in Turbulence X, Proc. of ETC10 Conference, Trondheim, Norway (Ed. : H.I. Anderson, P.-A. Krogstad, CIMNE, Barcelona, 2004)

43. O. Chanal, B. Chabaud, B. Castaing, B. Hébral, Eur. Phys. J. B 17, 309 (2000)

44. Y. Malécot, C. Auriault, H. Kahalerras, Y. Gagne, O. Chanal, B. Chabaud, B. Castaing, Eur. Phys. J. B 16, $549(2000)$

45. T. von Kármán, L. Howarth, Proc. Roy. Soc. A164 917 (1938)

46. A. Arneodo, C. Baudet, F. Belin, R. Benzi, B. Castaing, B. Chabaud, R. Chavarria, S. Ciliberto, R. Camussi, F. Chillà et al., Europhys. Lett. 34, 411 (1996)

47. E. Lindborg, Phys. of Fluids 11, 510 (1999)

48. L. Danaila, F. Anselmet, T. Zhou, R. Antonia, J. Fluid Mech. 391, 359 (1999)

49. F. Moisy, P. Tabeling, H. Willaime, Phys. Rev. Lett. 82, 3994 (1999)

50. T. Lundgren, Phys. of Fluids 14, 638 (2002)

51. Y. Gagne, B. Castaing, C. Baudet, Y. Malécot, Phys. of Fluids 16, 482 (2004)

52. N.R. Kevlahan, J. Alam, O. Vasilyev, J. Fluid Mech. 570, $217(2007)$

53. H. Tennekes, J. Lumley, A First Course in Turbulence (MIT Press, 1972)

54. I. Daubechies, Comm. Pure and App. Math. 41, 909 (1988)

55. P. Abry, P. Flandrin, M. Taqqu, D. Veitch, Wavelets for the analysis, estimation and synthesis of scaling data, in Self-similar Network Traffic and Performance Evaluation (Wiley, 2000)

56. P. Mattila, Geometry of sets and measures in Euclidean spaces (Cambridge University Press, 1995)

57. E. Novikov, Prikl. Math. Mekh. 35, 266 (1970) 


\begin{tabular}{ccccccc}
$R_{\lambda}$ & $\simeq 90$ & $\simeq 210$ & $\simeq 460$ & $\simeq 930$ & $\simeq 2000$ & $\simeq 2500$ \\
\hline velocity mean & $0.13 \mathrm{~m} \cdot \mathrm{s}^{-1}$ & $0.14 \mathrm{~m} \cdot \mathrm{s}^{-1}$ & $0.62 \mathrm{~m} \cdot \mathrm{s}^{-1}$ & $2.5 \mathrm{~m} \cdot \mathrm{s}^{-1}$ & $21 \mathrm{~m} \cdot \mathrm{s}^{-1}$ & $20 \mathrm{~m} \cdot \mathrm{s}^{-1}$ \\
velocity standard deviation & $0.024 \mathrm{~m} \cdot \mathrm{s}^{-1}$ & $0.032 \mathrm{~m} \cdot \mathrm{s}^{-1}$ & $0.13 \mathrm{~m} \cdot \mathrm{s}^{-1}$ & $0.51 \mathrm{~m} \cdot \mathrm{s}^{-1}$ & $1.7 \mathrm{~m} \cdot \mathrm{s}^{-1}$ & $1.9 \mathrm{~m} \cdot \mathrm{s}^{-1}$ \\
total duration (in integral scale) & $\simeq 96000$ & $\simeq 49000$ & $\simeq 29000$ & $\simeq 8000$ & $\simeq 2000$ & $\simeq 900$ \\
number of series & 3024 & 1532 & 892 & 248 & 64 & 27 \\
series duration (in integral scale) & $\simeq 32$ & $\simeq 32$ & $\simeq 32$ & $\simeq 32$ & $\simeq 32$ & $\simeq 32$
\end{tabular}

Table 1. Experimental data details.

\begin{tabular}{ccccc} 
model & $c_{1}$ & $c_{2}$ & $c_{3}$ & $c_{4}$ \\
\hline log-normal & $\simeq 0.371$ & -0.025 & 0 & 0 \\
She-Lévêquel & $\simeq 0.381$ & $\simeq-0.0365$ & $\simeq 0.00494$ & $\simeq-0.000667$
\end{tabular}

Table 2. Cumulant exponents of the log-normal (with the commonly accepted value: $c_{2}=-0.02$ ) and She-Lévêque models.

\begin{tabular}{cccccc}
$R_{\lambda}$ & $c_{1}^{l}$ & $c_{2}^{l}$ & $c_{3}^{l}$ & $c_{4}^{l}$ & $\zeta^{l}(3)$ \\
\hline$\simeq 930$ & $0.334 \pm 0.002$ & $-0.023 \pm 0.001$ & $0.0000 \pm 0.0003$ & $-0.0003 \pm 0.0002$ & $0.90 \pm 0.01$ \\
$\simeq 2000$ & $0.341 \pm 0.003$ & $-0.022 \pm 0.001$ & $-0.0001 \pm 0.0006$ & $-0.0002 \pm 0.0004$ & $0.92 \pm 0.01$ \\
$\simeq 2500$ & $0.349 \pm 0.004$ & $-0.024 \pm 0.002$ & $0.000 \pm 0.001$ & $0.000 \pm 0.001$ & $0.94 \pm 0.02$
\end{tabular}

Table 3. Cumulant exponents and third order scaling exponents computed with the WL.

\begin{tabular}{ccccc}
$R_{\lambda}$ & $\tilde{c}_{1}^{l}$ & $\tilde{c}_{2}^{l}$ & $\tilde{c}_{3}^{l}$ & $\tilde{c}_{4}^{l}$ \\
\hline$\simeq 930$ & $0.373 \pm 0.002$ & $-0.026 \pm 0.001$ & $0.0001 \pm 0.0003$ & $-0.0003 \pm 0.0002$ \\
$\simeq 2000$ & $0.370 \pm 0.003$ & $-0.024 \pm 0.002$ & $-0.000 \pm 0.001$ & $-0.0003 \pm 0.0004$ \\
$\simeq 2500$ & $0.373 \pm 0.004$ & $-0.026 \pm 0.002$ & $0.000 \pm 0.001$ & $0.000 \pm 0.001$
\end{tabular}

Table 4. Normalized cumulant exponents computed with the WL. 


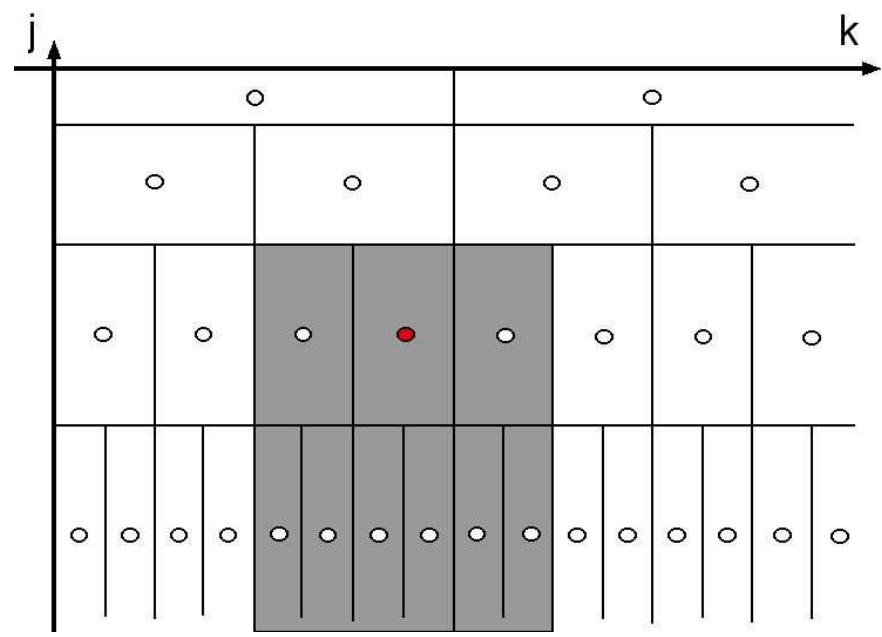

Fig. 1. Space-scale plane and the dyadic grid. Every dot (o) represents a discrete wavelet coefficient $d(j, k)$ and the surrounding rectangle the dyadic interval $\lambda(j, k)$. The shaded area sketches the subset $3 \lambda(j, k)$ associated to the wavelet leader $l(j, k)($ solid $\operatorname{dot} \bullet)$.
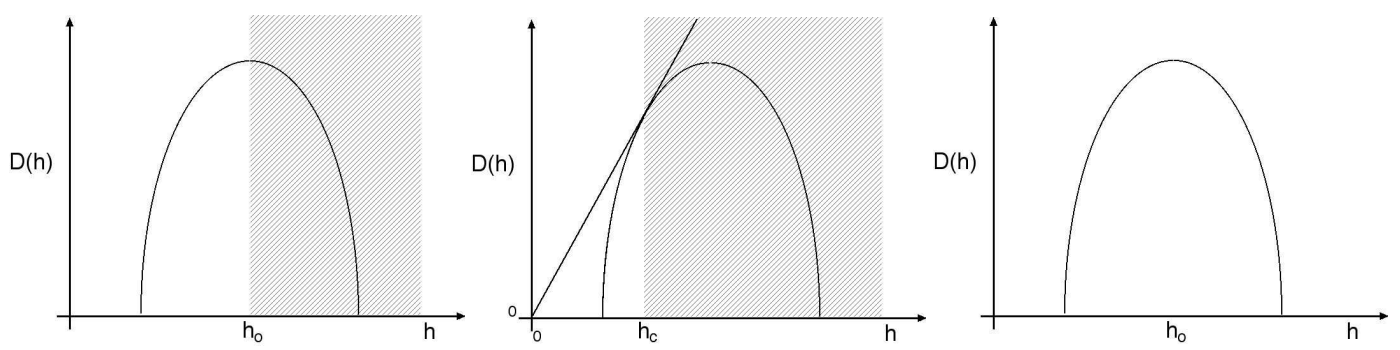

Fig. 2. Multifractal formalism validity. Domains of validity (white area) and of non-validity (dashed ares) of the DWC based MF formalism for uniform Hölder functions with only simple singularities (left), for all uniform Hölder functions (middle) and of the WL based MF formalism (right).
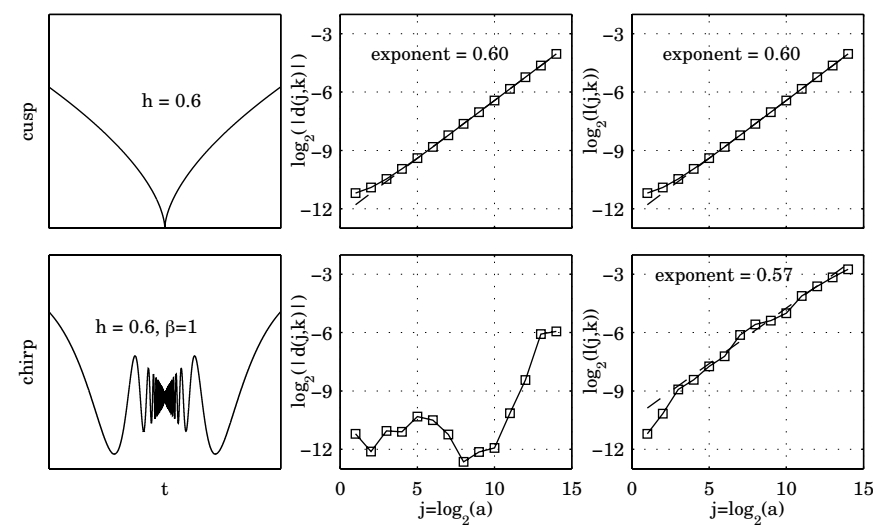

Fig. 3. Simple vs. oscillating singularities. DWC $d(j, k)$ and WL $l(j, k)$ computed with the cusp: $\left|x-x_{0}\right|^{0.6}$ (top) and with the chirp: $\left|x-x_{0}\right|^{0.6} \sin \left(\frac{1}{\left|x-x_{0}\right|^{1}}\right)$ (bottom) and plotted in a $\log _{2}-\log _{2}$ diagram $(k$ is chosen such that $0 \in \lambda(j, k))$. Daubechies wavelet with order 1 is used. 


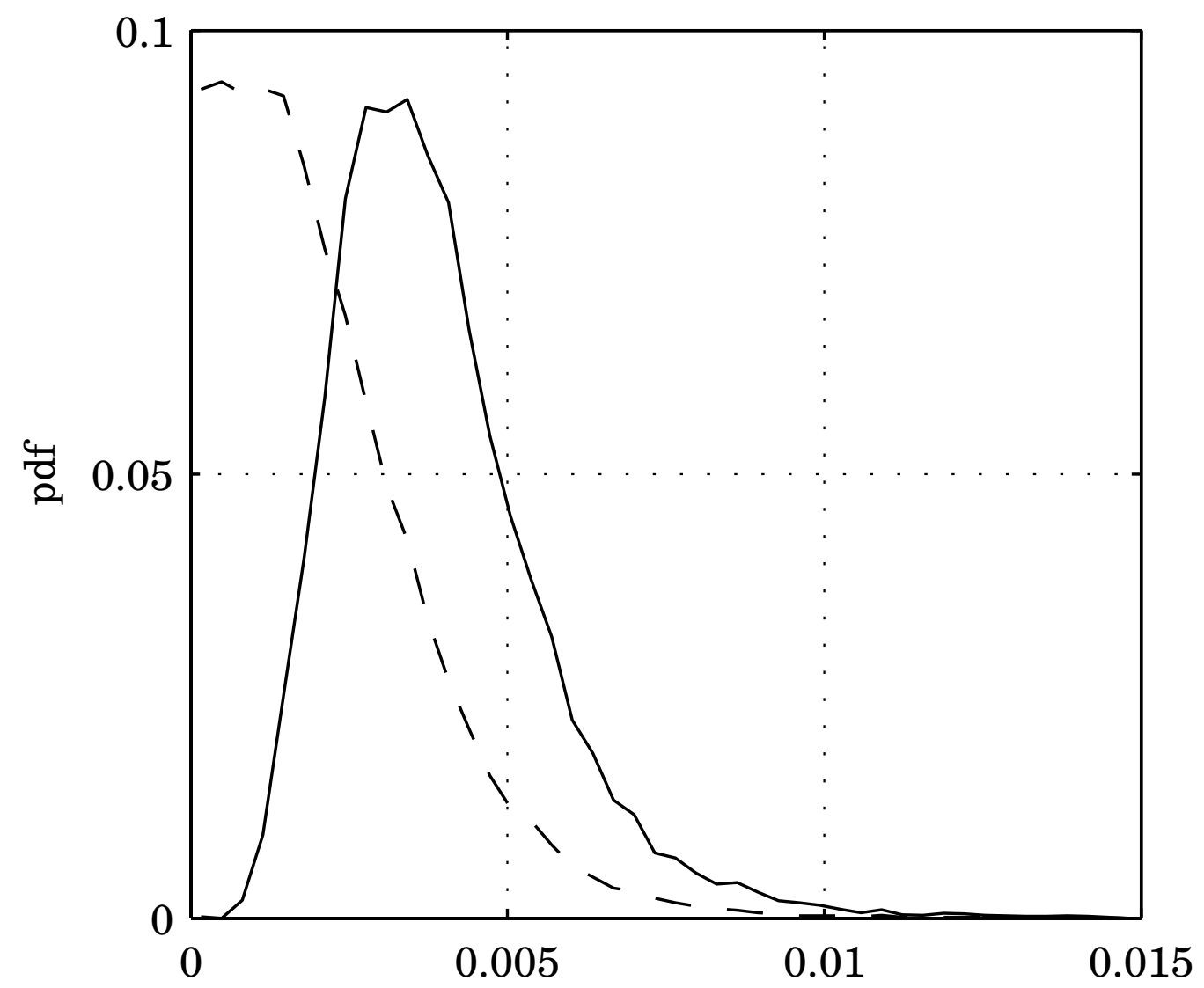

Fig. 4. Histograms of WL (solid line) and of absolute values of DWC (dashed line) computed on a realization of the random wavelet cascade. 

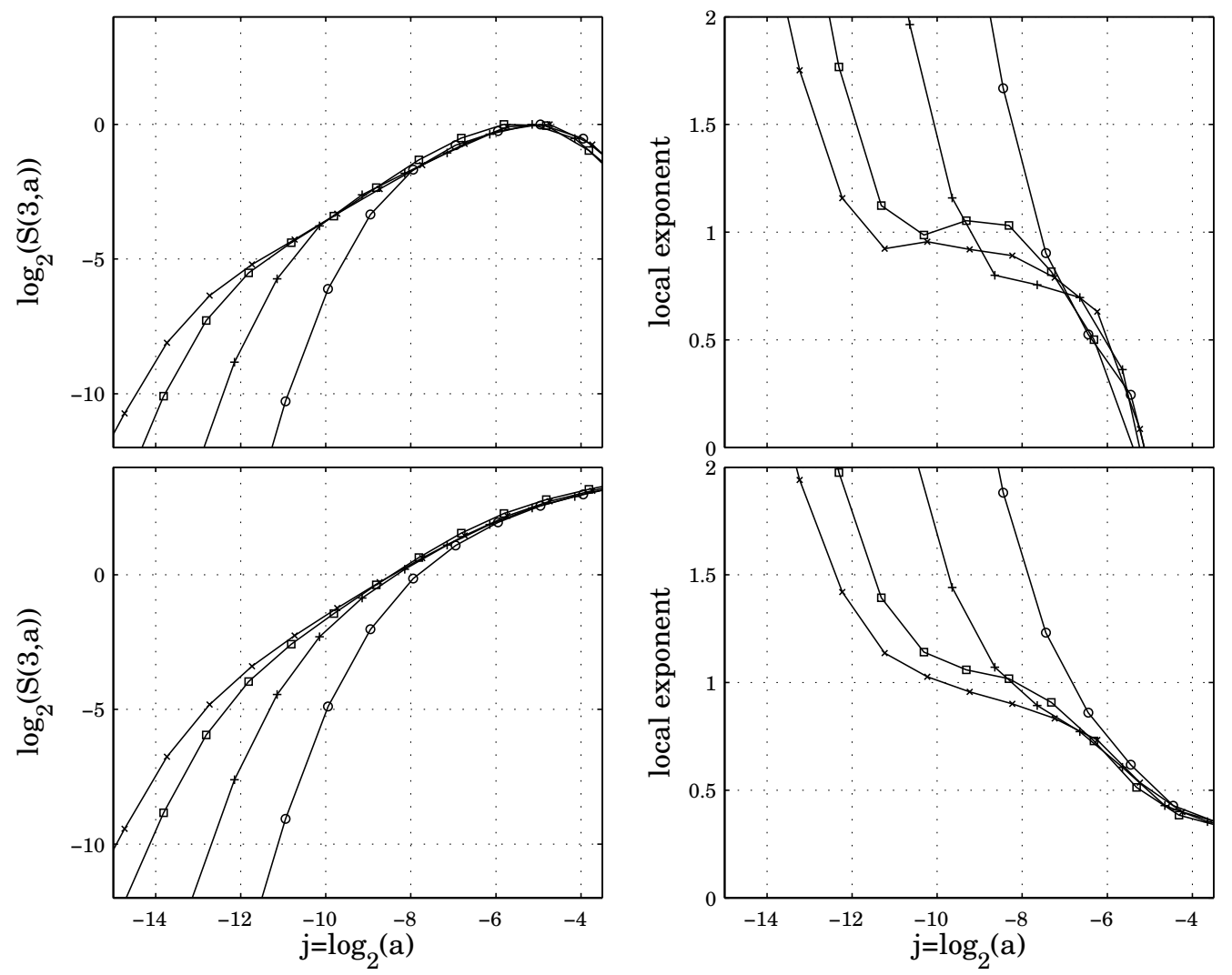

Fig. 5. Dependence of the third order structure function on $R_{\lambda}$. Third order structure functions (left) computed with DWC (top) and WL (bottom) and corresponding local exponent (right): $R_{\lambda} \simeq 90$ (o), $R_{\lambda} \simeq 210(+), R_{\lambda} \simeq 460(\square)$ and $R_{\lambda} \simeq 930(\times)$. The scale $a=2^{j}$ is in meters. 

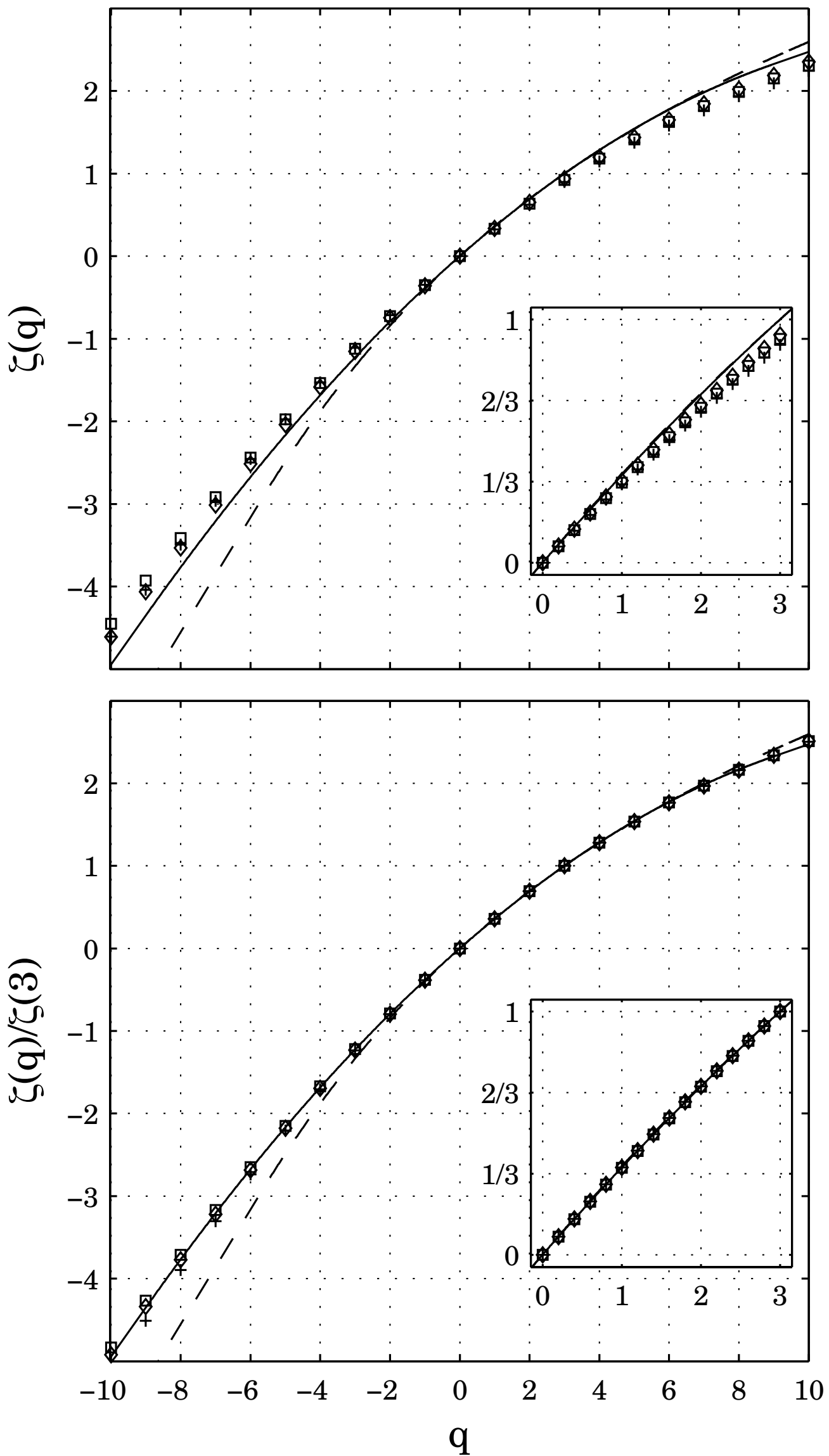

Fig. 6. Raw scaling exponents $\zeta^{l}(q)$ and normalized scaling exponents $\tilde{\zeta}^{l}(q)$. $R_{\lambda} \simeq 930(+), R_{\lambda} \simeq 2000(\square)$ and $R_{\lambda} \simeq 2500(\diamond)$. Log-normal (solid line) and She-Lévêque (dashed line) models are plotted. 

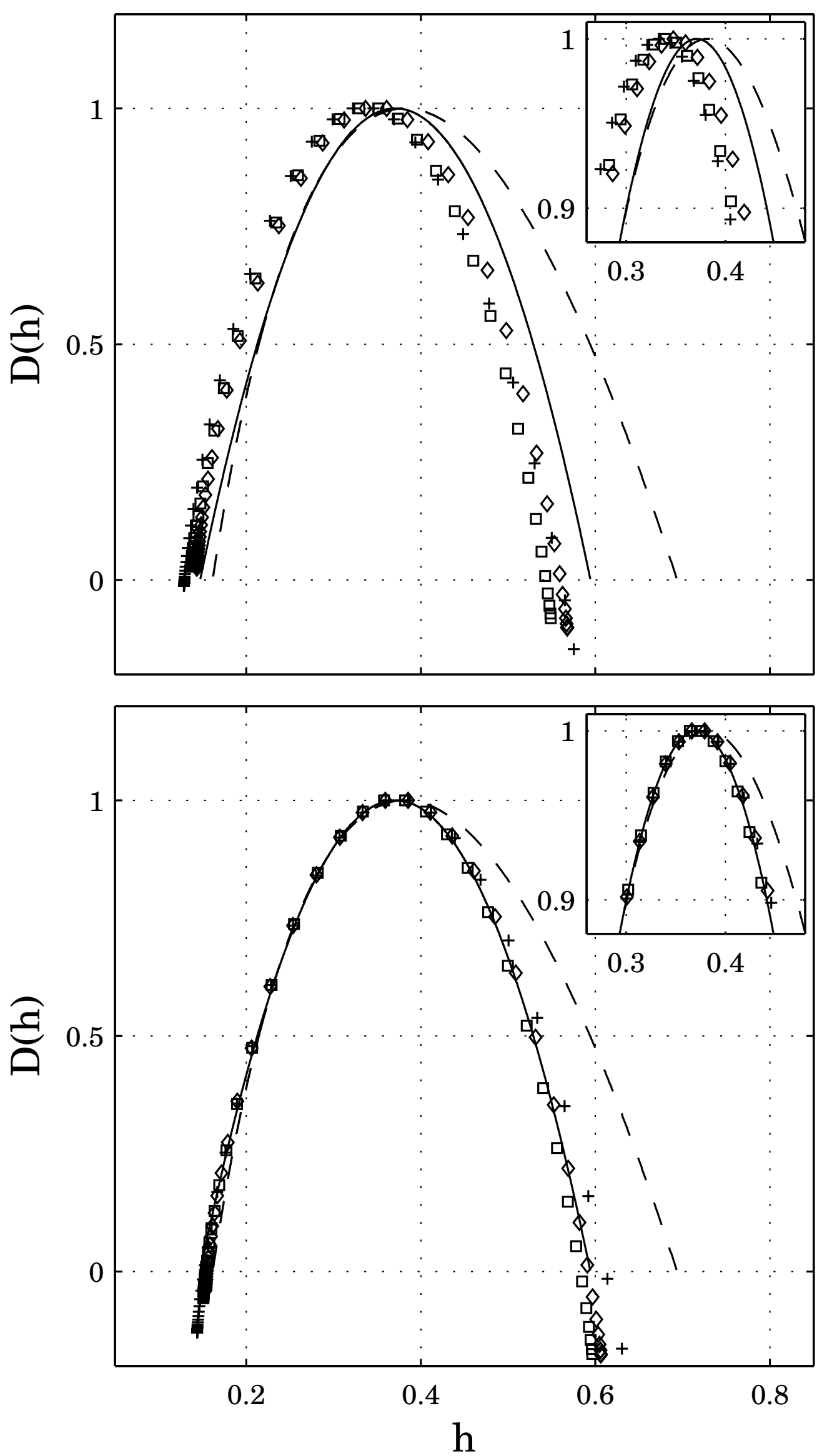

Fig. 7. Legendre transforms of the $\zeta^{l}(q)$ and the $\tilde{\zeta}^{l}(q) \cdot R_{\lambda} \simeq 930(+), R_{\lambda} \simeq 2000(\square)$ and $R_{\lambda} \simeq 2500(\diamond)$. Log-normal (solid line) and She-Lévêque (dashed line) models are plotted. 


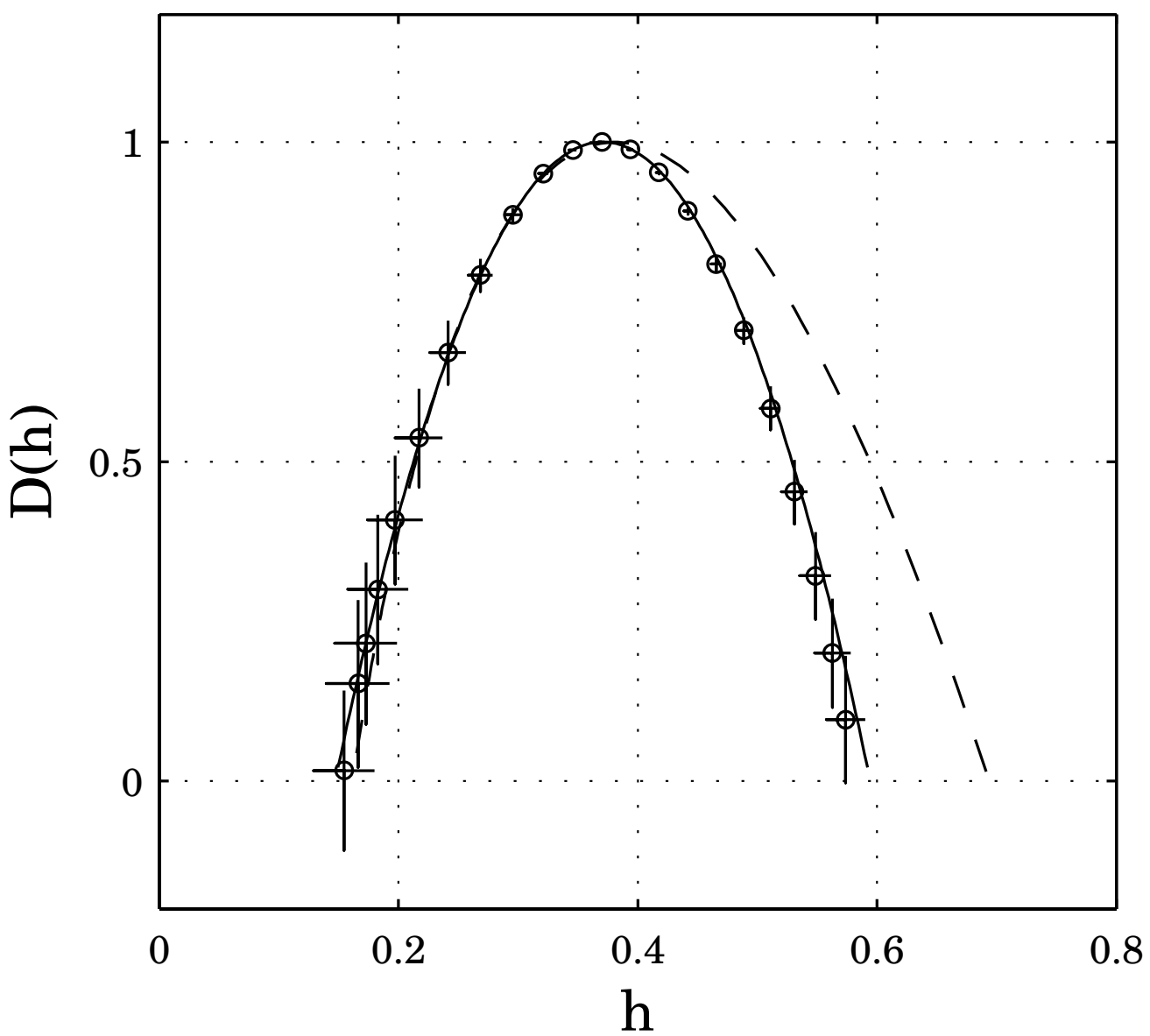

Fig. 8. $R_{\lambda} \simeq$ 2000. Singularity spectrum computed with WL (o) and computed confidence intervals. Log-normal (solid line) and She-Lévêque (dashed line) models are plotted. 

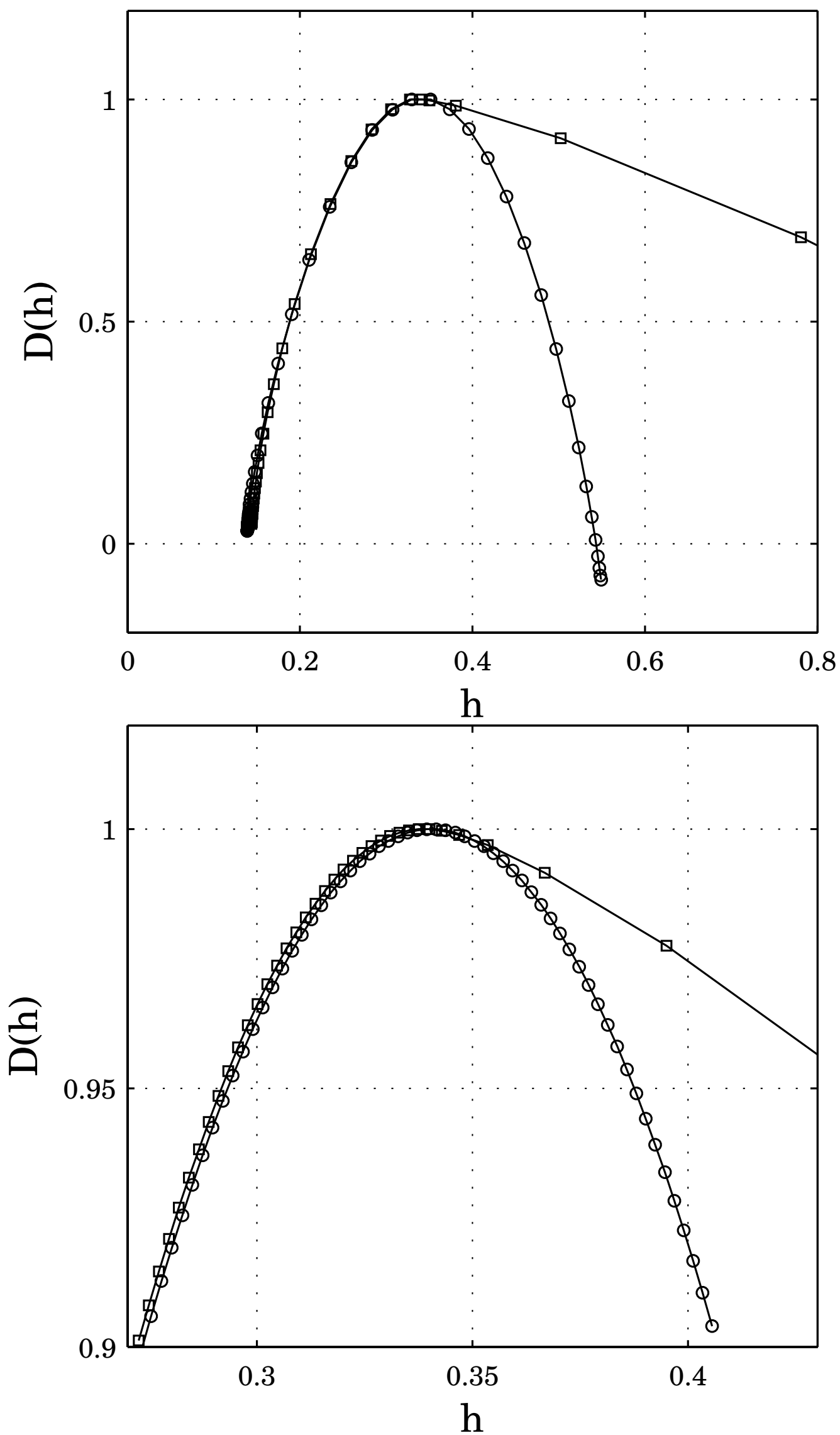

Fig. 9. $R_{\lambda} \simeq$ 2000. Singularity spectra computed with DWC ( $\square$ ) and WL (o). Top plot exhibits results for all $h$ values and bottom plot focuses in on $h$ values close to $h_{0}$ 

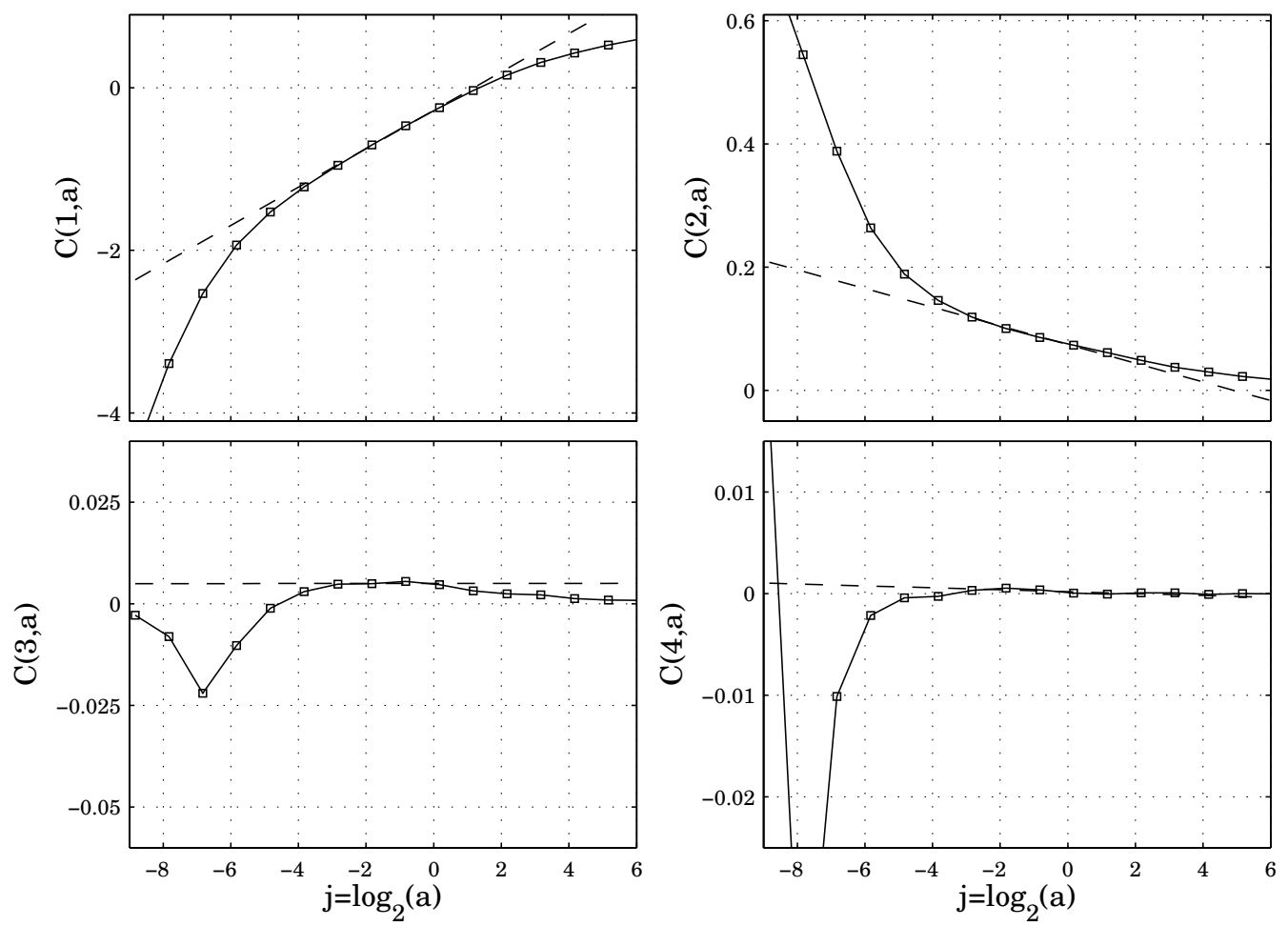

Fig. 10. $R_{\lambda} \simeq 2000$, cumulant analysis. $C^{l}(1, a)$ (top left), $C^{l}(2, a)$ (top right), $C^{l}(2, a)$ (bottom left) or $C^{l}(4, a)$. The scale $a=2^{j}$ is in meters. 